\title{
Pion quasiparticles and QCD phase transitions at finite temperature and isospin density from holography
}

\author{
Xuanmin Cao, ${ }^{*}$ Hui Liu $\odot{ }^{\dagger}$ and Danning Li $\odot^{*}$ \\ Department of Physics and Siyuan Laboratory, Jinan University, Guangzhou 510632, China
}

(Received 10 September 2020; accepted 17 November 2020; published 8 December 2020)

\begin{abstract}
The spectra of pions- known as the pseudo-Goldstone bosons of spontaneous chiral symmetry breaking - and their relationship with the chiral and pion superfluidity phase transitions have been investigated in the framework of soft-wall AdS/QCD. We prove that pions are massless Goldstone bosons in the chiral limit even at finite temperature, which was usually considered an assumption in soft-wall models. Above $T_{c}$, at which the chiral condensate $\langle\bar{q} q\rangle$ vanishes, the spectra of pions and scalar mesons merge, showing evidence of restored chiral symmetry at the hadronic spectrum level. Extending to finite quark mass, pion masses increase with quark mass. Further, it is more interesting to observe that the pole masses of pions decrease with temperature below $T_{c}$, which agrees with the analysis by Son and Stephanov [Phys. Rev. Lett. 88, 202302 (2002)]. Meanwhile, symmetry restoration above $T_{c}$ could be seen in the scalar and pseudoscalar meson spectra. With finite temperature and isospin chemical potential $\mu_{I}$, we show that the masses of charged pions are split. The mass of a positively charged pion $\pi^{+}$decreases almost linearly to zero when $\mu_{I}$ grows to its critical value $\mu_{I}^{c}$, where pion condensation begins. This reveals the Goldstone nature of $\pi^{+}$after the pion superfluidity transition, which is closely related to experimental observations.
\end{abstract}

DOI: $10.1103 /$ PhysRevD.102.126014

\section{INTRODUCTION}

Relativistic heavy-ion collisions (RHICs) provide an important approach to probe possible new states of nuclear matter in the laboratory [1]. The fireball created by collisions of high-energy nuclei is far from equilibrium, and it exists for only a few $\mathrm{fm} / c$, which makes it difficult to detect directly. Instead, the distributions and correlations of hadrons emitted after freeze-out and hadronization are the direct observables to probe the hot/dense nuclear matter. To understand the experimental data, the in-medium properties of particles are of critical importance. For example, the variation of meson mass at finite temperature might significantly change the final distribution of hadrons $[2,3]$. Therefore, it is quite essential to get a full understanding of the particles in the medium.

Among the different probes, the pion is an important and special one both theoretically and phenomenologically. It is the lightest meson, which shows its nature as a pseudo-

\footnotetext{
*caoxm@jnu.edu.cn

tliuhui@jnu.edu.cn

Corresponding author. lidanning@jnu.edu.cn
}

Published by the American Physical Society under the terms of the Creative Commons Attribution 4.0 International license. Further distribution of this work must maintain attribution to the author(s) and the published article's title, journal citation, and DOI. Funded by SCOAP ${ }^{3}$.
Goldstone boson. Consequently, it is closely related to the chiral phase transition, which happens between the broken and restored chiral symmetry phases. Thus, it is considered as a possible probe of the transition [4]. Furthermore, the detection of a significant fraction of coherent charged pions [5] at LHC energies suggests a possible superfluid phase consisting of condensed charged pions [6,7]. Besides, the ratio of the multiplicities of charged pions $\left(\pi^{-}\right.$and $\left.\pi^{+}\right)$ provides the possibility to extract nuclear symmetry energy, which plays an important role in neutron stars [8]. Thus, the properties of pions in the medium has attracted a lot of attention.

Due to its pseudo-Goldstone nature, the relevant physics of the pion spectrum in the medium is naturally nonperturbative. Actually, it is usually studied within nonperturbative frameworks, like lattice simulations [9-11], Dyson-Schwinger equations (DSEs) $[12,13]$, the functional renormalization group (FRG) $[14,15]$, and Nambu-JonaLasinio (NJL) models [16-21]. Generally, due to the effective interactions (attractive or repulsive) with the medium, pions might have different masses and widths at different temperatures and densities, and they were termed "quasipions" in Ref. [22]. At relatively high temperature, above the chiral transition temperature $T_{c}$, most of studies have obtained growing masses of pion quasiparticles with increasing temperature [12-14,17,18]. Such behavior might originate partly from the pions' Goldstone nature. In the chiral limit, $m_{\pi}$ remains zero at temperatures below $T_{c}$, and it can only increase with $T$ 
above $T_{c}$ (though the excitation modes might change). Then, extending to physical quark masses, it is quite reasonable to expect the increase of $m_{\pi}$ with temperature.. However, there are debates in the literature on the temperature behavior of $m_{\pi}$ with a physical quark mass below $T_{c}$. Son and Stephanov gave a general analysis in Refs. [23,24], and a decreasing $m_{\pi}$ below $T_{c}$ was proposed. An estimated $30 \%$ reduction of $m_{\pi}$ from its vacuum value to its value around $T_{c}$ was suggested. Qualitatively, this result was supported by lattice simulations in Refs. [10,11] and a study using an NJL model with gluon condensate in Ref. [16]. Nevertheless, other research groups using different methods-including NJL modes [17,18], FRG [14], lattice simulations [9], and DSEs [13]—obtained contrasting results, showing an increase of $m_{\pi}$ below $T_{c}$. A conclusion on this issue is still hard to be drawn, and more information from other approaches might be useful and necessary.

Fortunately, in recent decades the discovery of the AdS/CFT correspondence [25-27] offered another possibility to solve the strong-coupling problems of quantum chromodynamics (QCD). The shear viscosity to entropy density ratio $\eta / s$ was determined to be as small as $1 /(4 \pi) \approx 0.08$ [28], which agrees with the values used in fitting the elliptic flow $v_{2}$ of RHIC data [29-32]. In addition, the application of holographic methods in QCD has proven to be powerful in many other models, like top-down brane systems [33-37] and the bottom-up hard wall model [38], the soft-wall model [39], lightfront holographic QCD [40], Einstein-Maxwell-Dilaton systems [41-45], and so on (for a review, please refer to Refs. [46-50]).

Among these models, the hard-wall and soft-wall models provide a good starting point to describe the hadronic spectrum, as well as the chiral phase transition. The spectrum of pions in the vacuum extracted in their extended models agree very well with the experimental data [51-60]. Chiral symmetry breaking is well characterized by both a nonzero chiral condensate and the mass splits of chiral partners $\left[\left(\rho, a_{1}\right)\right.$ and $\left.\left(f_{0}, \pi\right)\right][51,52,55,56]$. Extended to finite temperature, it has been shown that the chiral condensate can be determined dynamically by the softwall model itself, and the chiral phase transition can be well described [61-71]. For pion quasiparticles at finite temperature, a few investigations have been made using extended hard-wall and soft-wall models [72-74]. Those studies showed that the pion mass decreases with temperature at a relatively low temperature. Nevertheless, as pointed out in Ref. [72], a constant chiral condensate is an input, and the restoration of chiral symmetry is neglected at high temperatures. Thus, the relationship between the spectrum of pion quasiparticles and the chiral phase transition in those models is still unknown. Besides, the high-temperature (above the chiral transition temperature $T_{c}$ ) behavior has not been investigated, and it is unclear whether chiral symmetry could be restored in the hadronic spectrum. Therefore, it is still interesting to investigate the pion spectrum at finite temperature in hard-wall and soft-wall models. From theoretical aspects, one should check whether $m_{\pi}$ remains zero below $T_{c}$ in the chiral limit to guarantee the Goldstone theorem $[75,76]$. Phenomenologically, moving to the realistic case with a physical quark mass, the temperaturedependent behavior of $m_{\pi}$ under the effect of a dynamically determined chiral condensate is still an interesting topic. Finally, it would be interesting to check whether chiral symmetry could be restored in the hadronic spectrum. It is meaningful to check whether a scalar meson $\sigma$ and pion could become degenerate above $T_{c}$. In this work, since the chiral condensate can be determined dynamically in soft-wall models, we consider all of these issues in the soft-wall framework. For the quasiparticle masses at finite temperature, we introduce the spectral method for our study, which has been applied throughout the literature to investigate QCD properties [77-89], and the masses are extracted from the locations of the peaks of the spectral functions.

Besides the temperature effects, density effects are of great interest as well. To seek the possible critical end point (CEP) in the $T-\mu_{B}$ (temperature-baryon number chemical potential) plane is the primary goal of the Beam Energy Scan (BES) project [90-92]. Recent experimental data also suggest the increasing effect of isospin density $n_{I}$ at LHC energies [5-7]. At large isospin chemical potential $\mu_{I}$, a transition from the normal phase to a pion superfluidity phase, consisting of condensed charged pions, might play an important role at this energy scale. Thus, there is growing interest in the density-dependent behavior of the pion mass. In the bottom-up holographic framework, the pure isospin density effect has been studied in the hard-wall model [93-97]. A transition from a normal phase to a pion condensed phase has been shown to occur at $\mu_{I}=m_{\pi}$, $T=0$. Also, the mass splitting of mesons has been shown in the hard-wall model. Since those studies focused on finite isospin density only and the mutual effect from temperature is unclear, we extend those studies to finite temperature in the soft-wall model and get the $T-\mu_{I}$ phase diagram [98,99]. Here, we also continue our studies and consider the mutual effect of isospin densities and temperature on pion quasiparticles.

The paper is organized as follows. In Sec. II, after a brief review of the chiral phase transition in the soft-wall model, we extract the mass spectra of pion quasiparticles and scalar mesons from spectral functions at finite temperature. In the chiral limit, both numerically and analytically, we prove the Goldstone nature of pions at low temperatures. An extension of the Gell-Mann-Oakes-Renner (GOR) relation at finite temperature and physical quark mass is given. In Sec. III we study the mutual effect of temperature and isospin density on the pion spectrum. In Sec. IV we give our conclusions. 


\section{QUASIPIONS AND THE CHIRAL PHASE TRANSITION AT FINITE TEMPERATURE IN A SOFT-WALL MODEL}

As mentioned above, both the hard-wall and soft-wall models provide a good starting point to deal with the hadronic spectrum and the chiral phase transition. Incorporating the global symmetry of QCD, it could be naturally extended to cases with multiple flavors [67,70,71], finite temperature [61-71], finite baryon number density $\mu_{B}[61,70]$, finite isospin number density $\mu_{I}$ $[98,99]$, different space-time dimensions [100,101], and so on. Since the soft-wall model can be imposed on the radial excitations and the equation of motion can self-consistently determine the chiral condensate, the soft-wall model provides a better framework for studying the relationship between the spectrum and phase transition. Here, we briefly review the soft-wall model.

The model starts from an action with $S U_{L}\left(N_{f}\right) \times$ $S U_{R}\left(N_{f}\right)$ gauge symmetry, which reads ${ }^{1}$

$$
\begin{aligned}
S= & \int d^{5} x \sqrt{g} e^{-\Phi(z)} \operatorname{Tr}\left\{\left|D_{M} X\right|^{2}-V_{X}(|X|)\right. \\
& \left.-\frac{1}{4 g_{5}^{2}}\left(F_{L}^{2}+F_{R}^{2}\right)\right\} .
\end{aligned}
$$

Here, $g$ is the determinant of the background metric $g_{M N}$; $\Phi(z)$ is the dilaton field, which depends only on the fifth dimension $z ; X X$ is the matrix-valued bulk field, which is dual to the operator $\bar{q}^{\alpha} q^{\beta}$, where $\alpha, \beta$ are the indices in flavor space; $V_{X}=m_{5}^{2}|X|^{2}+\lambda|X|^{4}$ is the potential of $X$, where $m_{5}^{2}$ is the five-dimensional (5D) mass of $X$ and $\lambda$ is a free parameter of the potential; and $g_{5}$ is the gauge coupling, which can be determined to be $g_{5}=2 \pi$ by comparing the large-momentum expansion of vector current correlator $\left[J_{\mu}^{a}=\bar{q} \gamma_{\mu} t^{a} q, \mu=(0,1,2,3)\right]$ to the perturbation calculation [38]. In this work, we focus on $N_{f}=2$, with the lightest two flavors. Thus, $t^{a}(a=1,2,3)$ are taken as the generators of $S U(2)$. The covariant derivative $D_{M} X$ with $M=(\mu, 5)$ and the gauge field strengths $F_{M N}^{L / R}$ are defined as

$$
\begin{gathered}
D_{M} X=\partial_{M} X-i L_{M} X+i X R_{M}, \\
F_{M N}^{L}=\partial_{M} L_{N}-\partial_{N} L_{M}-i\left[L_{M}, L_{N}\right], \\
F_{M N}^{R}=\partial_{M} R_{N}-\partial_{N} R_{M}-i\left[R_{M}, R_{N}\right],
\end{gathered}
$$

where the gauge fields $L_{M}=L_{M}^{a} t^{a}, R_{M}=R_{M}^{a} t^{a}$ are dual to left- and right-handed currents in the four-dimensional (4D) field theory at the boundary, i.e., $L_{\mu}^{a} \leftrightarrow \bar{q}_{L} \gamma_{\mu} t^{a} q_{L}$ and $R_{\mu}^{a} \leftrightarrow$

\footnotetext{
${ }^{1}$ If one considers the baryon number density, a $U(1)$ related to the $U(1)_{B}$ symmetry can be added.
}

$\bar{q}_{R} \gamma_{\mu} t^{a} q_{R}$ [38]. For later convenience, one can redefine the chiral gauge fields as the vector gauge field and the axialvector gauge field,

$$
\begin{aligned}
& V_{M}=\frac{L_{M}+R_{M}}{2}, \\
& A_{M}=\frac{L_{M}-R_{M}}{2},
\end{aligned}
$$

where the vector $V_{M}^{a}$ and the axial field $A_{M}^{a}$ correspond to the vector and axial-vector current, $J_{V \mu}^{a}$ and $J_{A \mu}^{a}$, respectively. After a transformation, the covariant derivative and the transformed gauge field strength are deformed as

$$
\begin{gathered}
D_{M} X=\partial_{M} X-i\left[V_{M}, X\right]-i\left[A_{M}, X\right], \\
F_{M N}^{V}=\frac{1}{2}\left(F_{M N}^{L}+F_{M N}^{R}\right), \\
F_{M N}^{A}=\frac{1}{2}\left(F_{M N}^{L}-F_{M N}^{R}\right) .
\end{gathered}
$$

In this section, we focus on the temperature effect on scalar and pseudoscalar mesons. Thus, all of the gauge fields will be set to zero. In the QCD vacuum, only the diagonal components of the operator $\bar{q}^{\alpha} q^{\beta}$ have nonvanishing expectation values. Accordingly, on the dual gravity side, $X$ would be taken as

$$
X=\chi t^{0},
$$

where we have $t^{0}=I_{2} / 2$, where $I_{2}$ a $2 \times 2$ is the identity matrix. Up to now, if one couples the soft-wall action (1) with certain gravity systems, like the Einstein-MaxwellDilaton systems [41-45], one can solve the background gravity and extract the information for the 4D field theory. However, the full numerical process is difficult. Here, we follow previous studies [61-71] and take Eq. (1) as a probe. In this way, the profile of the dilaton field, the background geometric would be considered as an input generated from a certain Einstein-Maxwell-Dilaton system. ${ }^{2}$ Since the AdS-Schwarzchild black hole solution has been widely tested, we follow those studies and take the geometric as ${ }^{3}$

$$
d s^{2}=e^{2 A(z)}\left(f(z) d t^{2}-d x^{i} d x_{i}-\frac{1}{f(z)} d z^{2}\right),
$$

where $z_{h}$ is the horizon of the black hole and $z \leq z_{h}$ [46]. Therefore, the integration interval of the fifth dimension is

\footnotetext{
${ }^{2}$ Generally, one might solve the background field from a fixed dilaton potential like in Refs. [41-45,102-104] or use the potential reconstruction approach to construct such a background like in Refs. [105-110].

${ }^{3}$ In the current work, the topology of the boundary is flat and the AdS radius $L$ will be cancelled everywhere. Thus, we have set $L=1$.
} 
always $\left(0, z_{h}\right)$, which is exactly outside the black hole. Nevertheless, one can also have the fifth dimension in the interval of $\left(z_{h}, \infty\right)$ by an inverse transformation. Besides,

$$
\begin{aligned}
& A(z)=-\ln (z), \\
& f(z)=1-\frac{z^{4}}{z_{h}^{4}} .
\end{aligned}
$$

The temperature is encoded by the following equation:

$$
T=\left|\frac{f^{\prime}\left(z_{h}\right)}{4 \pi}\right|=\frac{1}{\pi z_{h}} .
$$

In the original soft-wall model, the dilaton field is taken as

$$
\Phi(z)=\mu_{g}^{2} z^{2}
$$

where $\mu_{g}$ is a free parameter, which will be determined from the meson spectrum.

The 5D mass $m_{5}$ is determined via the relation [26,27] $m_{5}^{2}=\Delta(\Delta-4)$, where $\Delta=3$ is the dimension of the scalar operator $\bar{q}_{R}^{\alpha} q_{L}^{\beta}$ in the original hard-wall and soft-wall models $[38,39]$. However, for the field $X$ in the nonconformal field theory, a $z$-dependent correction can be incorporated. It effectively introduces the anomalous dimension $\gamma\left(\mu_{r}\right)$ by replacing $\Delta$ with $\Delta+\gamma\left(\mu_{r}\right)$ [42]. If one maps the renormalization scale $\mu_{r}$ to the $5 \mathrm{D} z$, then $m_{5}^{2}$ would be naturally a function of $z$ [111,112]. Phenomenologically, it has been shown that there is no spontaneous chiral symmetry breaking under such a dilaton and constant 5D mass $m_{5}^{2}$. Either modifying the dilaton field $[63,64]$ or modifying the $5 \mathrm{D}$ mass [69] is necessary. In Ref. [69], to match the experimental data of the meson spectra, it was found that $\chi(z)$ should behave as $m_{q} z+\frac{\sigma}{\zeta} z^{3}+\cdots$ at the UV boundary and be linear in $z$ at the IR boundary. Thus, the $z$-dependent $m_{5}^{2}(z)$ is determined to be the sum of a constant term and a quadratic term, ${ }^{4}$

$$
m_{5}^{2}(z)=-3-\mu_{c} z^{2}
$$

where $\mu_{c}$ is another free parameter. According to the study in Ref. [69],

$\mu_{g}=440 \mathrm{MeV}, \quad \mu_{c}=1450 \mathrm{MeV}, \quad \lambda=80$

gives the best fit of the meson spectra. Thus, we take this group of parameters in the following calculation. It should be pointed out that the main goal of this paper is to investigate the trends of the pion and scalar meson. Therefore, the analysis of this paper is mainly qualitative.

\footnotetext{
${ }^{4}$ Actually, one can also consider it as a modification of the interaction between the scalar $\chi$ and dilaton $\phi$.
}

Inserting Eqs. (5)-(10) into Eq. (1), one can easily derive the equation of motion for $\chi$ as

$$
\begin{aligned}
\chi^{\prime \prime} & +\left(3 A^{\prime}+\frac{f^{\prime}}{f}-\Phi^{\prime}\right) \chi^{\prime}+\frac{e^{2 A}}{f} \\
& \times\left[\left(3+\mu_{c}^{2} z^{2}\right)-\frac{\lambda \chi^{2}}{2}\right] \chi=0 .
\end{aligned}
$$

The above equation has two boundaries: the ultraviolet $(z=0)$ and the infrared $\left(z=z_{h}\right)$. The asymptotic behaviors at the two boundaries can be obtained as

$$
\begin{aligned}
\chi(z \rightarrow 0)= & m_{q} \zeta z+\frac{m_{q} \zeta}{4}\left(-2 \mu_{c}^{2}+4 \mu_{g}^{2}+m_{q}^{2} \zeta^{2} \lambda\right) \\
& \times z^{3} \ln (z)+\frac{\sigma}{\zeta} z^{3}+\mathcal{O}\left(z^{4}\right), \\
\chi\left(z \rightarrow z_{h}\right)= & c_{0}+\frac{c_{0}\left(2 \mu_{c}^{2} z_{h}^{2}-c_{0}^{2} \lambda+6\right)}{8 z_{h}-4 \gamma \mu^{2} z_{h}^{3}}\left(z-z_{h}\right) \\
& +\mathcal{O}\left[\left(z-z_{h}\right)^{2}\right],
\end{aligned}
$$

where $m_{q}$ and $\sigma$ are the two independent integral constants in the UV, dual to the quark mass and chiral condensate, respectively. $\zeta$ is a normalization constant and it was determined to be $\zeta=\sqrt{N_{c}} / 2 \pi$ in Ref. [113]. Here, we take $N_{c}=3$ for realistic QCD. $c_{0}$ is the integral constant generating a regular solution at the horizon $z_{h}$, while the other integral constant in the IR is dropped since it leads to a divergent solution at $z_{h}$. It is interesting to observe that, with a fixed quark mass $m_{q}$, one can solve for $\sigma$ and $c_{0}$ simultaneously from Eq. (13) with the "shooting method" introduced in the Appendix.

\section{A. The chiral phase transition in the soft-wall model}

Taking the values of the parameters in Eq. (12), one can obtain the temperature- and quark-mass-dependent behaviors of the chiral condensate. The chiral phase transition reflects the breaking of the chiral symmetry $S U(2) \times$ $S U(2)$, which is the exact symmetry in the 4D theory only in the chiral limit, with vanishing quark masses, as a finite quark mass would always generate a slight breaking of the symmetry. As a theoretical check, one should check whether symmetry breaking appears in this limit first. Therefore, we take $m_{q}=0$ in Eq. (14) and extract $\sigma$ at different temperatures. The result is shown by the black line in Fig. 1(a). From the figure, we can see that $\sigma \approx$ $(0.246 \mathrm{GeV})^{3}=0.0148 \mathrm{GeV}^{3} \neq 0$ at temperatures near zero. Thus, spontaneous chiral symmetry breaking is realized at low temperature. We can also see that $\sigma$ decreases with $T$ and it vanishes at $T_{c}=0.163 \mathrm{GeV}$. Above $T_{c}, \sigma$ becomes zero and chiral symmetry is restored. Actually, from the study in Ref. [114], the existence of $T_{c}$ is governed by the linearized equation 

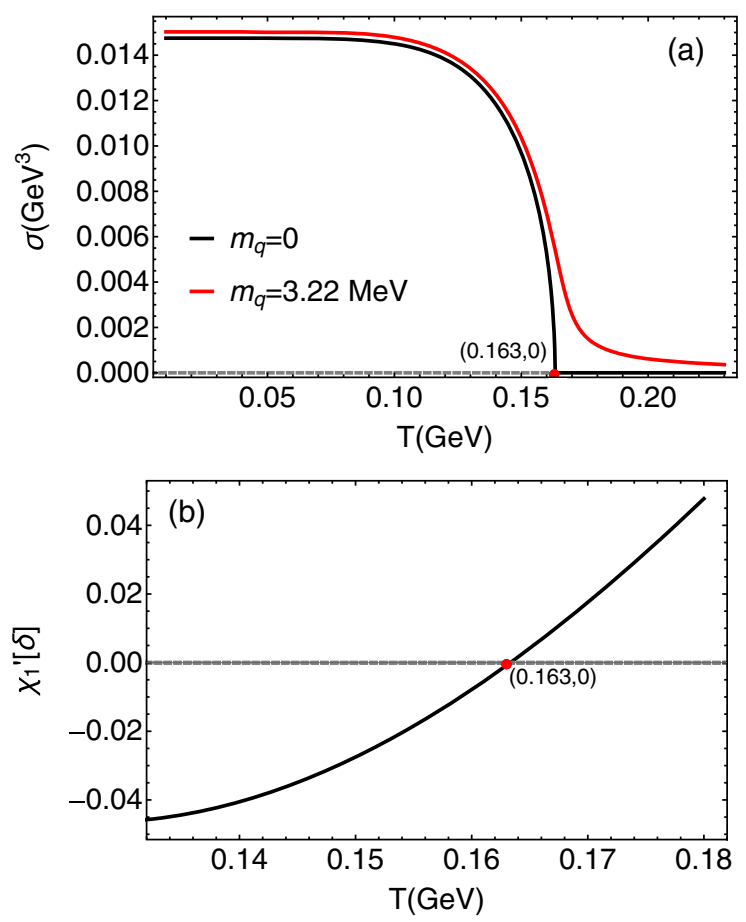

FIG. 1. (a) Temperature-dependent behavior of the chiral condensate. The black line stands for the chiral limit, $m_{q}=0$, where the critical temperature is labeled with a red solid dot, $T_{c}=0.163 \mathrm{GeV}$. The red line stands for $m_{q}=3.22 \mathrm{MeV}$ with pseudocritical temperature $T_{c p}=0.164 \mathrm{GeV}$ [69]. (b) Temperature-dependent behavior of $\chi_{1}^{\prime}(\delta)$ from Eq. (15), where $\delta$ is a vanishingly small constant and is set to $10^{-8} \mathrm{GeV}^{-1}$ in our numerical calculations. The red dot, where $\chi_{1}^{\prime}(\delta)=0$, is located at $(T=0.163 \mathrm{GeV}, 0)$.

$\chi_{1}^{\prime \prime}+\left(3 A^{\prime}+\frac{f^{\prime}}{f}-\Phi^{\prime}\right) \chi_{1}^{\prime}+\frac{e^{2 A}}{f}\left(3+\mu_{c}^{2} z^{2}\right) \chi_{1}=0$,

which is the expansion at $T_{c}$ around the leading solution $\chi_{0} \equiv 0$. The existence of $T_{c}$ requires the existence of a solution to the above equation, with the boundary condition

$$
\chi_{1}^{\prime}(z=0)=0, \quad \chi_{1}^{\prime}\left(z=z_{h}\right)=\text { finite } .
$$

This conclusion was proved in Ref. [114]. Here, as a numerical check, we take $\chi_{1}\left(z_{h}\right)=1$ and solve Eq. (15) numerically. Then, we plot $\chi^{\prime}(z=0)$ as a function of $T$ in Fig. 1. It is easy to see that $T_{c}$ in Fig. 1 is located exactly at the temperature where $\chi_{1}^{\prime}(z=0)=0$. So we conclude that at $T_{c}$, Eq. (15) has a solution satisfying $\chi_{1}^{\prime}(z=0)=0$ and $\chi_{1}^{\prime}\left(z=z_{h}\right)=$ finite.

After the theoretical check in the chiral limit, it is also important to move to the case with a physical quark mass. According to Ref. [69], $m_{q}=3.22 \mathrm{MeV}$ gives the best fit of experimental data. Thus, we take this value of the quark mass and solve Eq. (13) to obtain the temperaturedependent $\sigma(T)$. The result is shown by the red line in Fig. 1(a). From this figure, we can see that the secondorder phase transition turns out to be a crossover-type transition. Due to the finite quark mass, the exact chiral symmetry at the 4D Lagrangian level is explicitly broken, though only slightly. Thus, the phase transition is weakened. Furthermore, one can extract the pseudocritical temperature $T_{c p}=0.164 \mathrm{GeV}$ where $\left|\sigma^{\prime}(T)\right|$ reaches its maximum.

\section{B. Spectral functions for scalar and pseudoscalar modes at finite temperature}

In the previous section the phase transition was well described by the order parameter $\sigma$. As mentioned in the previous section, this is also expected to be seen from the hadron spectrum. In the chiral limit, as considering pion as the Goldstone bosons of the symmetry breaking below $T_{c}$, one might expect the pion mass $m_{\pi}$ to remain zero at any temperature below $T_{c}$. Also, since the scalar meson $f_{0}$ or the $\sigma$ meson is the chiral partner of the pion, one might ask whether they would become degenerate at a temperature above $T_{c}$. Therefore, we will try to study the temperature behavior of the pion and scalar meson. The particles are excitations of the vacuum in the 4D theory. In the dual description, they are perturbations around the background field on the gravity side. Thus, to describe the pion and scalar meson, we should consider the following perturbations:

$$
X=(\chi+S) t^{0} e^{2 i \pi^{a} t^{a}},
$$

where $\pi^{a}$ and $S$ are the pseudoscalar and scalar perturbations, respectively. In addition, one can check that the pseudoscalar perturbation will couple with the longitudinal $\operatorname{part}\left(\varphi^{i}\right)$ of the axial vector

$$
\begin{gathered}
a_{\mu}^{i}=a_{\mu}^{T, i}+\partial_{\mu} \varphi^{i}, \\
\partial^{\mu} a_{\mu}^{T, i}=0 .
\end{gathered}
$$

Thus, $\varphi^{i}$ should be considered as well.

Inserting the above perturbation into the action (1) and keeping the quadratic terms, one gets the effective part for the scalar perturbation as

$$
\begin{aligned}
S_{S}= & \frac{1}{2} \int d x^{5} \sqrt{g} e^{-\Phi}\left[g^{\mu \nu} \partial_{\mu} S \partial_{\nu} S+g^{z z}\left(\partial_{z} S\right)^{2}-m_{5}^{2} S^{2}\right. \\
& \left.-\frac{3 \lambda}{2} \chi^{2}(S)^{2}\right],
\end{aligned}
$$

and that for the pseudoscalar part as 


$$
\begin{aligned}
S_{P S}= & -\frac{1}{2 g_{5}^{2}} \int d^{5} x \sqrt{g} e^{-\Phi} \sum_{i=1}^{3}\left\{g^{\mu \nu} g^{z z} \partial_{z} \partial_{\mu} \varphi^{i} \partial_{z} \partial_{\nu} \varphi^{i}\right. \\
& -g_{5}^{2} \chi^{2}\left(g^{\mu \nu} \partial_{\mu} \varphi^{i} \partial_{\nu} \varphi^{i}+g^{\mu \nu} \partial_{\mu} \pi^{i} \partial_{\nu} \pi^{i}\right. \\
& \left.\left.+g^{z z}\left(\partial_{z} \pi^{i}\right)^{2}-2 g^{\mu \nu} \partial_{\mu} \varphi^{i} \partial_{\nu} \pi^{i}\right)\right\} .
\end{aligned}
$$

Generally, $S$ and $\pi^{i}, \varphi^{i}$ are functions of all of the coordinates. Here, since we focus on the quasiparticle's pole mass at finite temperature, we assume that all of the perturbations only depend on $t$ and $z$. Thus, one can transform the perturbations to frequency space by the Fourier transformations

$$
S(t, z)=\frac{1}{2 \pi} \int d \omega e^{-i \omega t} S(\omega, z)
$$

and

$$
\begin{aligned}
& \pi^{i}(t, z)=\frac{1}{2 \pi} \int d \omega e^{-i \omega t} \pi^{i}(\omega, z), \\
& \varphi^{i}(t, z)=\frac{1}{2 \pi} \int d \omega e^{-i \omega t} \varphi^{i}(\omega, z) .
\end{aligned}
$$

Under these conditions, one can get the equation of motion for the scalar quasiparticle as

$S^{\prime \prime}+\left(3 A^{\prime}+\frac{f^{\prime}}{f}-\Phi^{\prime}\right) S^{\prime}+\left(\frac{\omega^{2}}{f^{2}}-\frac{2 m_{5}^{2}+3 \lambda \chi^{2}}{2 f} A^{\prime 2}\right) S=0$,

and that for the pseudoscalar quasiparticle as ${ }^{5}$

$$
\begin{array}{r}
\varphi^{\prime \prime}+\left(A^{\prime}-\Phi^{\prime}\right) \varphi^{\prime}-\frac{e^{2 A} g_{5}^{2} \chi^{2}}{f}(\varphi-\pi)=0, \\
\pi^{\prime \prime}+\left(3 A^{\prime}+\frac{f^{\prime}}{f}-\Phi^{\prime}+\frac{2 \chi^{\prime}}{\chi}\right) \pi^{\prime}-\frac{(\varphi-\pi) \omega^{2}}{f^{2}}=0 .
\end{array}
$$

Here, we have neglected the isospin index $i$ in $\pi^{i}, \varphi^{i}$ due to the isospin symmetry at finite temperature.

Due to the interaction with the hot medium, the spectra of particles are broadening at finite temperature, and the good description is the spectral function. The quasiexcitation appears as the peak of the spectral function. The spectral function can be extracted from the retarded Green function $G^{R}(\omega)$,

$$
\rho(\omega)=-\frac{1}{\pi} \operatorname{Im} G^{R}(\omega) .
$$

\footnotetext{
${ }^{5}$ We note that the coupling to $\varphi$ is neglected in Refs. [73,74], which is different from our scenario.
}

The holographic correspondence states that the 4D operator $\mathcal{O}(x)$ and 5D field $\phi(x, z)$ are connected through the relation between the 4D generating function with an external source $\phi_{0}(x)$ and the classical action $S_{5 \mathrm{D}}$ in AdS space as

$$
\left\langle e^{i \int d^{4} \phi_{0}(x) \mathcal{O}(x)}\right\rangle_{\mathrm{CFT}}=\left.e^{i S_{\mathrm{5D}}\left[\phi^{c l}\right]}\right|_{\phi^{c l}(x, z=0)=\phi_{0}},
$$

where $\phi^{c l}$ is the classical solution of $S_{5 \mathrm{D}}\left[\phi^{c l}\right]$ with its boundary value equaling the external source $\phi_{0}(x)$ [26-28]. Therefore, the Green functions can be obtained by differentiating the 5D effective action with respect to the external sources. Here, for the scalar mode, the on-shell action becomes

$S_{S}^{\mathrm{on}}=-\left.\frac{1}{2} \int d \omega f(z) S(-\omega, z) e^{3 A(z)-\Phi(z)} S^{\prime}(\omega, z)\right|_{z=\epsilon} ^{z=z_{h}}$,

where $\epsilon$ is a UV cutoff. For the pseudoscalar mode, the onshell action has the following form:

$$
\begin{aligned}
S_{\pi}^{\mathrm{on}}= & -\frac{1}{2 g_{5}^{2}} \int d \omega e^{A-\Phi}\left[e^{2 A} g_{5}^{2} f \chi^{2} \pi(-\omega, z) \pi^{\prime}(\omega, z)\right. \\
& \left.-\omega^{2} \varphi(-\omega, z) \varphi^{\prime}(\omega, z)\right]\left.\right|_{z=\epsilon} ^{z=z_{h}} .
\end{aligned}
$$

Up to now, the main task has been to solve $S, \pi, \varphi$ from Eqs. (24) and (25a)-(25b). For the scalar mode, from Eq. (24), one can get the UV asymptotic solution of $S$ as

$$
\begin{aligned}
S(z \rightarrow 0)= & s_{1} z+s_{3} z^{3}-\frac{1}{4} s_{1} z^{3}\left[2 \mu_{c}^{2}-4 \mu_{g}^{2}-3 \zeta^{2} \lambda m_{q}^{2}\right. \\
& \left.+2 \omega^{2}\right] \log (z)+\mathcal{O}\left(z^{3}\right) .
\end{aligned}
$$

Here, $s_{1}$ and $s_{3}$ are the two integral constants of the secondorder ordinary derivative equation (ODE). From the holographic principle, we assume that $s_{1}$ corresponds to the external source, while $s_{3}$ corresponds to the scalar operator $\bar{q} q$. Inserting this expansion into the on-shell action (28), one gets the retarded Green function and spectral function of the scalar mode as

$$
\begin{gathered}
G_{S}^{R}(\omega)=\left.\frac{\delta^{2} S_{S}^{\text {on }}}{\delta s_{1}^{*} \delta s_{1}}\right|_{z=\epsilon}, \\
\rho_{S}(\omega)=-\frac{1}{\pi} \operatorname{Im} G_{S}^{R}(\omega) \\
=\frac{2}{\pi} \operatorname{Im}\left[\frac{s_{3}}{s_{1}}\right],
\end{gathered}
$$

Here, since we focus on the imaginary part of the Green function, we have erased the real part inside the "Im" function. Right now, both $s_{1}$ and $s_{3}$ are free integral 
constants. The retarded Green function property of Eq. (31) is related to the IR boundary at the horizon $z_{h}$. In fact, the $\frac{\omega^{2}}{f^{2}}$ leads to the infalling and outgoing boundary conditions at the horizon. According to the study in Ref. [115], to get the retarded Green function one has to impose the infalling boundary condition. Then, we have

$$
S\left(z \rightarrow z_{h}\right) \sim\left(z_{h}-z\right)^{-i \omega / 4 \pi T} .
$$

Taking this boundary condition and solving the equation of motion (24), one can obtain $s_{1}, s_{3}$, and the spectral function.

Similarly, for the pseudoscalar mode, from Eqs. (25a)(25b) one can obtain the asymptotic expansion at the UV boundary as

$\varphi(z \rightarrow 0)=c_{f}-\frac{1}{2} \zeta^{2} g_{5}^{2} m_{q}^{2} \pi_{0} z^{2} \log (z)+\varphi_{2} z^{2}+\mathcal{O}\left(z^{3}\right)$,

$\pi(z \rightarrow 0)=\pi_{0}+c_{f}-\frac{1}{2} \pi_{0} \omega^{2} z^{2} \log (z)+\pi_{2} z^{2}+\mathcal{O}\left(z^{3}\right)$,

where $c_{f}, \varphi_{2}, \pi_{0}$, and $\pi_{2}$ are the four integral constants of the two second-order ODEs. We note that Eqs. (25a)-(25b) are invariant under the transformations $\pi \rightarrow \pi+c_{f}$ and $\varphi \rightarrow \varphi+c_{f}$. Thus, it is easy to understand that $c_{f}$ corresponds to a redundant degree of freedom, and we will set it to zero. Then, only the three integral constants $\pi_{0}, \varphi_{2}$, and $\pi_{2}$ are relevant. Substituting the asymptotic solutions (34a)-(34b) into Eq. (29), one gets

$$
\begin{aligned}
S_{\pi}^{\text {on }}= & \int d \omega\left[\frac{1}{4} \pi_{0}^{*} \zeta^{2} m_{q}^{2}\left(\pi_{0} \omega^{2}-4 \pi_{2}\right)\right. \\
& \left.+\frac{1}{2} \zeta^{2} m_{q}^{2} \omega^{2}\left|\pi_{0}\right|^{2} \log (z)\right]\left.\right|_{z=\epsilon} ^{z=z_{h}} .
\end{aligned}
$$

According to the holographic dictionary, the coupled 5D fields of $\varphi$ and $\pi$ can be decomposed in terms of bulk-toboundary propagators $[116,117]$ as

$$
\begin{aligned}
& \varphi(\omega, z)=\frac{i J(\omega)}{\omega} \tilde{\varphi}(\omega, z), \\
& \pi(\omega, z)=\frac{i J(\omega)}{\omega} \tilde{\pi}(\omega, z),
\end{aligned}
$$

where $J(\omega)$ is the external source of the longitudinal component of the axial current operator $J_{A \mu}^{0}$, or the value of the $\varphi$ field on the boundary. Comparing to Eqs. (34a)(34b), one can identify the boundary value $\pi_{0}$ as the source $J(\omega)$. Then, the retarded Green function can be extracted from the second derivative of the action with respect to the source. From Eq. (35), it reads

$$
\begin{aligned}
G_{\pi}^{R}(\omega) & =\left.\frac{\delta^{2} S_{\pi}^{\text {on }}}{\delta \pi_{0}^{*} \delta \pi_{0}}\right|_{z=\epsilon} \\
& =\frac{1}{4 \pi_{0}} \zeta^{2} m_{q}^{2}\left(\pi_{0} \omega^{2}-4 \pi_{2}\right)+\left.\frac{1}{2} \zeta^{2} m_{q}^{2} \omega^{2} \log (z)\right|_{z=\epsilon} .
\end{aligned}
$$

Taking the relation in Eq. (26), one can get the spectral function of the pion as

$$
\rho_{\pi}(\omega)=-\frac{1}{\pi} \operatorname{Im} G_{\pi}^{R}(\omega)=\frac{m_{q}^{2} \zeta^{2}}{\pi} \operatorname{Im}\left[\frac{\pi_{2}}{\pi_{0}}\right] .
$$

As in the scalar mode, to get the retarded Green function one has to impose the infalling boundary condition at $z_{h}$. On this side, the asymptotic expansion reads

$$
\begin{aligned}
\varphi\left(z \rightarrow z_{h}\right)= & \left(z_{h}-z\right)^{-\frac{i \omega z_{h}}{4}}\left\{-\frac{4 i c_{0}^{2} g_{5}^{2} \pi_{\mathrm{h} 0}\left(z-z_{h}\right)}{\omega z_{h}^{2}\left(i \omega z_{h}-4\right)}\right. \\
& \left.+\mathcal{O}\left[\left(z-z_{h}\right)^{2}\right]\right\}+c_{h 0}, \\
\pi\left(z \rightarrow z_{h}\right)= & \left(z_{h}-z\right)^{-\frac{i \omega z_{h}}{4}}\left\{\pi_{h 0}+\mathcal{O}\left(z-z_{h}\right)\right\}+c_{h 0} .
\end{aligned}
$$

Here, $\pi_{h 0}$ and $c_{h 0}$ are the two integral constants describing the infalling mode, while another two describing the outgoing mode are omitted. The parameter $c_{0}$ is the integral constant as shown in Eq. (14). Since the ODEs are linear, one can take $\pi_{h 0}=1$ without shifting the spectra. The other integral constant $c_{h 0}$, as well as the integral constants in the $\mathrm{UV} \pi_{0}, \varphi_{2}$, and $\pi_{2}$, can be determined by requiring $c_{f}=0$ when applying the "shooting" algorithm. Then, one obtains the spectral function for the pseudoscalar mode. After all of these preparations, we will extract and discuss the spectral functions and the spectra of the scalar and pseudoscalar quasiparticles in the next three subsections.

\section{The chiral limit: Goldstone bosons and symmetry restoration of chiral partners}

As mentioned above, theoretically, it is important to check whether the pseudoscalar mode at finite temperature is the Goldstone mode. If it is not, there would be theoretical inconsistency in the soft-wall model. To check this, the quark mass $m_{q}$ is set to zero, i.e., in the chiral limit, to guarantee the exact chiral symmetry in the 4D Lagrangian. Then, we solve $\chi$ by applying the process described in Sec. II A. By substituting $\chi$ into the process described in Sec. II B, one can obtain the spectral functions for both the scalar and pseudoscalar modes. We take $T=0.060,0.120$, and $0.150 \mathrm{GeV}$ as an example and plot the spectral functions in Figs. 2(a) and 2(b). We can see that there are peaks in the spectral functions. These peaks are related to thermal excitations. Thus, we can extract the masses from the locations of these peaks. From Fig. 2(b), 

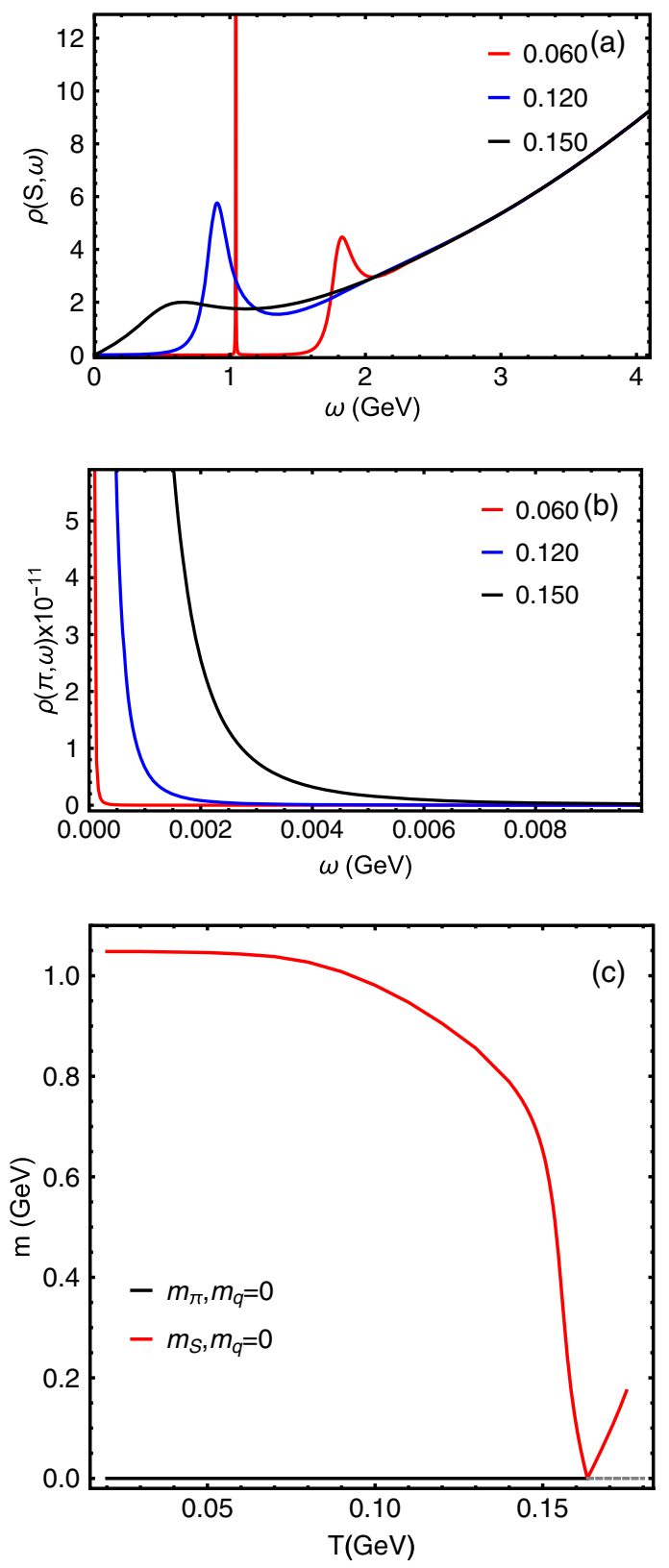

FIG. 2. Spectral functions in frequency space of (a) the scalar meson $\rho(S, \omega)$ and (b) the pion $\rho(\pi, \omega)$, in the chiral limit at $T=0.06,0.12$, and $0.15 \mathrm{GeV}$. (c) Temperature dependence of the lowest $m_{S}$ and $m_{\pi}$ in the chiral limit.

we can see that for the pseudoscalar mode, below $T_{c}$, there is always a divergence located at $\omega=0$, which reveals $m_{\pi}=0$. The temperature-dependent behaviors of $m_{\pi}$ and $m_{S}$ are shown in Fig. 2(c). From the figure, we can see that $m_{S}$ is about $1.05 \mathrm{GeV}$ near $T=0$. It decreases at low temperature and reaches zero at $T=0.163 \mathrm{GeV}$, which is exactly the chiral transition temperature $T_{c}$ in the chiral limit. Moreover, it is interesting to observe that, above $T_{c}$, $m_{\pi}$ and $m_{S}$ converge to the same line and increase together very fast. Numerically, in the soft-wall model the
Goldstone nature of the pion is checked, and chiral symmetry restoration is realized at the hadronic level.

Actually, this conclusion can also be proved analytically. First, it is quite easy to understand why $m_{S}=0$ at $T_{c}$ in the chiral limit. We have proved in Sec. II A that, at and above $T_{c}, \chi \equiv 0$ in the chiral limit. Substituting this equation into Eq. (24) and setting $\omega=0$, it is easy to see that Eq. (24) becomes the same as the $T_{c}$ criteria (15). In fact, the solution of Eq. (15) under the boundary condition (16) is just the wave function of a massless scalar mode at $T_{c}$. Physically, in the chiral limit, when $m_{S}=0$, instability appears. Actually, this is the physical meaning of the $T_{c}$ criteria (15).

Then, since $\chi \equiv 0$ above $T_{c}$, from Eq. (17) the coupling between $\pi$ and $\varphi$ disappears. Since the modulus of $X$ vanishes, the expansion of $X$ can no longer be Eq. (17). Instead, the scalar expansion and the pseudoscalar expansion should have the same form. As a result, the masses of the two modes are naturally the same. In this sense, the red piece above $T_{c}$ in Fig. 2(c) is for $m_{\pi}$ as well, though it is actually obtained from the scalar sector.

Finally, we will try to get an analytical understanding of the masses of pions below $T_{c}$. As we have shown, below $T_{c}$ the pseudoscalar mode satisfies Eqs. (25a)-(25b). Our main goal is to check the existence of the massless mode with $\omega=0$. Under this condition, we see that Eq. (25b) becomes

$$
\pi^{\prime \prime}+\left(3 A^{\prime}+\frac{f^{\prime}}{f}-\Phi^{\prime}+\frac{2 \chi^{\prime}}{\chi}\right) \pi^{\prime}=0,
$$

which can be directly solved as

$$
\pi(z)=p_{1}+p_{2} \int_{0}^{z} \frac{e^{-3 A+\Phi}}{f \chi^{2}} d z^{\prime}
$$

where $p_{1}$ and $p_{2}$ are the two integral constants of the second-order ODE. However, the $p_{2}$ branch is not physically acceptable at either the UV or IR boundary.

At the UV boundary $z=0$, in the chiral limit, the leading expansions are $\chi \sim z^{3}, f \sim 1, e^{-3 A}=z^{3}$, and $e^{\Phi} \sim 1$. Thus, the integral kernel is divergent as $z^{-3}$. Only when quark mass is finite, this part could appear in the wave function.

At the horizon $z=z_{h}$, the leading expansions are $\chi \sim$ const, $f \sim\left(z-z_{h}\right), e^{-3 A}=$ const, $\quad$ and $\quad e^{\Phi} \sim$ const, and it is also divergent. Therefore, the physically acceptable solution for $\pi$ is $\pi \equiv$ const. Since we will normalize $\pi_{0}=1$ in calculating spectral functions, the constant should be chosen as 1 . Thus, the existence of the massless mode is equivalent to the existence of a solution to the equation [subject to $\varphi(0)=0$ ]

$$
\varphi^{\prime \prime}+\left(A^{\prime}-\Phi^{\prime}\right) \varphi^{\prime}-\frac{e^{2 A} g_{5}^{2} \chi^{2}}{f}(\varphi-1)=0,
$$

which comes from Eq. (25b) by replacing $\pi$ with 1 . Redefining $\tilde{\varphi}=\varphi-1$, one reaches a linear ODE, 


$$
\tilde{\varphi}^{\prime \prime}+\left(A^{\prime}-\Phi^{\prime}\right) \tilde{\varphi}^{\prime}-\frac{e^{2 A} g_{5}^{2} \chi^{2}}{f} \tilde{\varphi}=0
$$

The asymptotic expansion of the above second-order ODE can be easily extracted as

$$
\tilde{\varphi}=p_{0}+p_{2} z^{2}+\mathcal{O}\left(z^{4}\right)
$$

at the UV boundary and

$$
\begin{aligned}
\tilde{\varphi}= & p_{h 0}\left(1+\frac{g_{5}^{2} \chi\left(z_{h}\right)^{2}}{4 z_{h}}\left(z-z_{h}\right) \ln \left(z_{h}-z\right)\right) \\
& +p_{h 1}\left(z-z_{h}\right)+\mathcal{O}\left(\left(z-z_{h}\right)^{2}\right)
\end{aligned}
$$

at the IR boundary, where $p_{0}, p_{2}, p_{h 0}$, and $p_{h 1}$ are the corresponding integral constants on both sides. The $p_{h 0}$ branch should be dropped since it leads to a divergent $\varphi^{\prime}$ at $z_{h}$. Thus, only $p_{h 1}$ can be nonzero. Considering the linearity of the equation, one can take $p_{h 1}=1$ and solve for $\tilde{\varphi}$ using the equation. After one gets the solution, one can normalize $p_{0}$ to 1 , again from the equation's linearity. Then, one has actually obtained the massless wave function $\varphi=\tilde{\varphi}+1$ and $\pi \equiv 1$ subject to the boundary condition $\varphi(0)=0$. Therefore, we have proved that the massless mode always exists below $T_{c}$, and it is the Goldstone mode or the massless pion at finite temperature. It should be pointed out that the above proof cannot be extended to finite quark mass. The reason is that with finite quark mass, there would be terms $\frac{p_{0} g_{5}^{2} m^{2} \zeta^{2}}{2} z^{2} \ln (z)$ between $p_{0}$ and $p_{2} z^{2}$, which leads to a divergent on-shell action. So it is not dual to the physical mode. As a result, with a finite quark mass, the massless mode does not exist for most of the cases in the soft-wall model. ${ }^{6}$ Also, it cannot be extended to temperatures above $T_{c}$. When $\chi \equiv 0$ above $T_{c}$, the equations of motion for pseudoscalar and scalar modes should be the same, and the two kinds of excitations would become degenerate.

To summarize, we have proved that the pion is always massless in the chiral limit by numerical calculation and analytical analysis. It is the Goldstone mode of the spontaneous symmetry breaking below $T_{c}$. The analytical proof is quite general for most of the models in the soft-wall AdS/QCD framework. Moreover, the expected degeneration in spectral of chiral partners is observed.

\section{Physical quark mass: Pole masses of quasipions}

In the previous section we checked the theoretical consistency of the soft-wall model in the chiral limit. To

\footnotetext{
${ }^{6}$ Of course, if one chooses the background field properly, with finite quark masses, the massless mode might appear at a certain temperature. However, it is still hard to guarantee the existence of a Goldstone mode at any temperature below $T_{c}$. We have checked that in the current model, there will not be such a weird result.
}

be more realistic and to get more information about the current experimental data, we consider the situation with a physical quark mass, $m_{q}=3.22 \mathrm{MeV}$. With a finite quark mass, below $T_{c}$, the pion might gain a certain mass due to the explicit chiral symmetry breaking.

Taking $m_{q}=3.22 \mathrm{MeV}$ and the values of the parameters in Eq. (12), one can solve for $\chi$ using Eq. (13). Then, by imposing the IR boundary condition (33) and Eqs. (39a)(39b) and solving Eqs. (24) and (25a)-(25b), and after normalizing $s_{1}=1$ and $\pi_{0}=1$, one can obtain $s_{3}, \varphi_{2}$, and $\pi_{2}$, which are defined in the UV expansion (30) and (34a)-(34b). Inserting these results into the expressions for the spectral functions in Eqs. (31), (37), and (38), one can obtain the finite-temperature spectral functions for the scalar meson and pion.

From the numerical calculation, we find that the behavior of the spectral function at temperatures below the pseudotransition temperature $T_{c p}=0.164 \mathrm{GeV}$ [Fig. 1(a)] is different from that at temperatures above $T_{c p}$. To show this difference, we take $T=0.070,0.140,0.160 \mathrm{GeV}$ and $0.166,0.170,0.175 \mathrm{GeV}$ and extract the spectral functions for the scalar meson and pion in Fig. 3. The left two panels are the results of the scalar spectral functions, while the right two panels are for the pseudoscalar spectral functions. The upper two panels give the low-temperature behavior. At very low $T$, e.g., $T=0.07 \mathrm{GeV}$ (the red lines in the upper two panels), for th scalar and pseudoscalar spectral functions there are sharp peaks at around $\omega=1.05 \mathrm{GeV}$ and $\omega=0.137 \mathrm{GeV}$, respectively, very close to the vacuum values of the scalar and pseudoscalar mesons. To the right of the first peaks, at around $\omega=1.88 \mathrm{GeV}$ for the scalar function and $\omega=1.52 \mathrm{GeV}$ for the pseudoscalar function, wide peaks corresponding to the radial excitations at zero temperature appear in the two spectral functions. When the temperature increases to $T=0.14 \mathrm{GeV}$, we can see that the centers and heights of the left peaks decrease, while the widths of the peaks are broadened (though still quite sharp). This shows the decrease of the masses of the scalar meson and pion at low temperatures. Moreover, the right peaks at $T=0.14 \mathrm{GeV}$ cannot be identified at this temperature due to the rapid decrease of the heights and the rapid increase of the widths. This might correspond to the melting of the higher excitations at finite temperature. Actually, this is quite reasonable. It is easier to destroy the weaker binding of the higher excitations, so they melt before the ground states. When the temperature increases to $T=0.16 \mathrm{GeV}$ (still slightly lower than $T_{c p}$ ) we find that the masses continue to decrease, while the peaks become very wide. Thus, we can see the decrease of the meson mass at temperatures below $T_{c p}$, which is similar to the decrease of the masses in the chiral limit. In some sense, the chiral limit behavior also governs the small quark masses' behavior. The chiral condensate and meson mass results with small quark masses differ slightly from that in the chiral limit. 

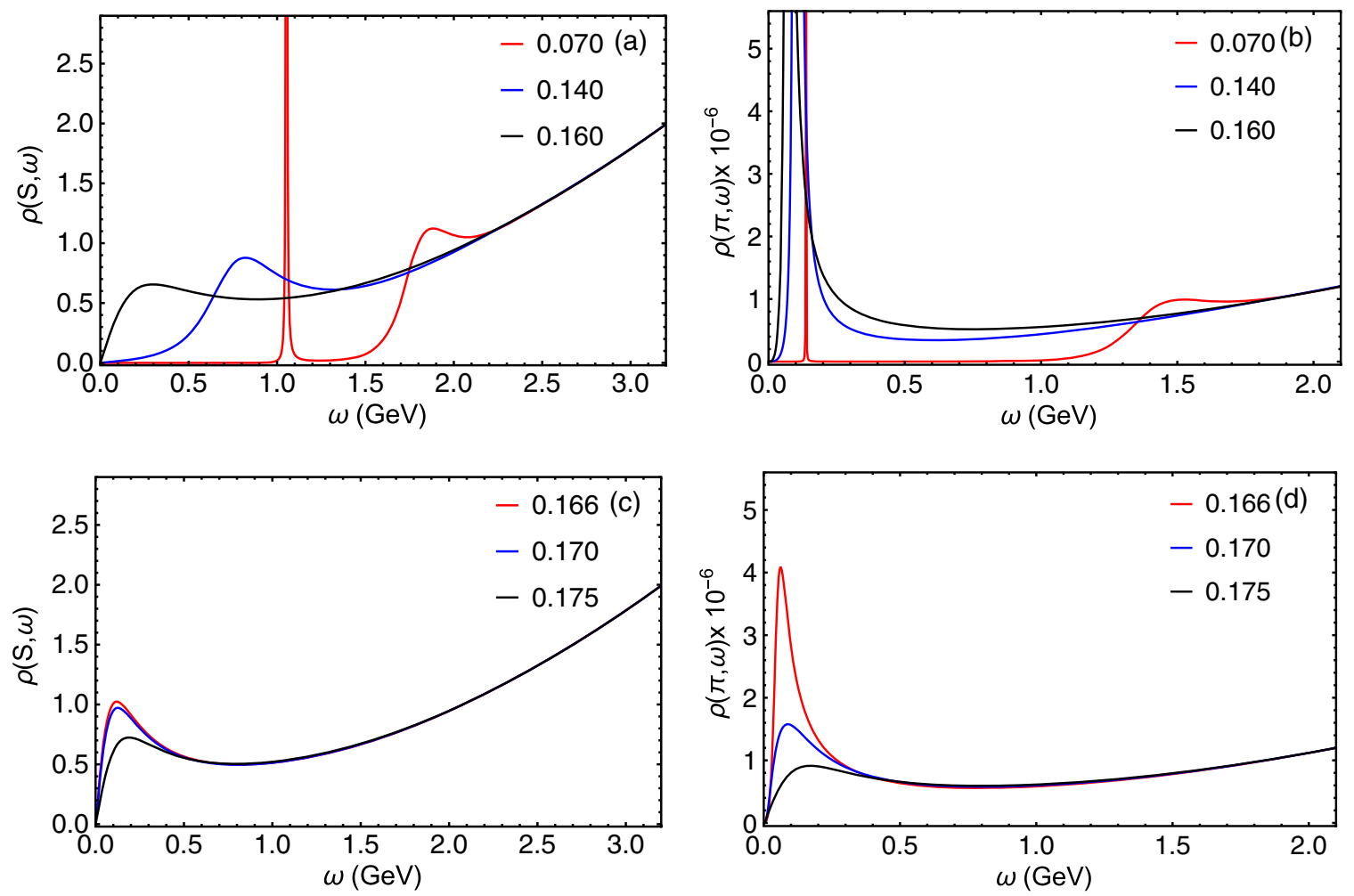

FIG. 3. Spectral functions of (a) the scalar mode $\rho(S, \omega)$ at $T=0.070,0.140$, and $0.160 \mathrm{GeV}$, (b) the pion $\rho(\pi, \omega)$ at $T=0.070,0.140$, and $0.160 \mathrm{GeV}$, (c) the scalar mode $\rho(S, \omega)$ at $T=0.166,0.170$, and $0.175 \mathrm{GeV}$, and (d) the pion $\rho(\pi, \omega)$ at $T=0.166,0.170$, and $0.175 \mathrm{GeV}$.

Then, we increase the temperature further. From the lower two panels in Fig. 3, we can see that at temperatures above $T_{c p}$ (but not far away from $T_{c p}$ ) the left peaks are still alive. However, the temperature behaviors are totally

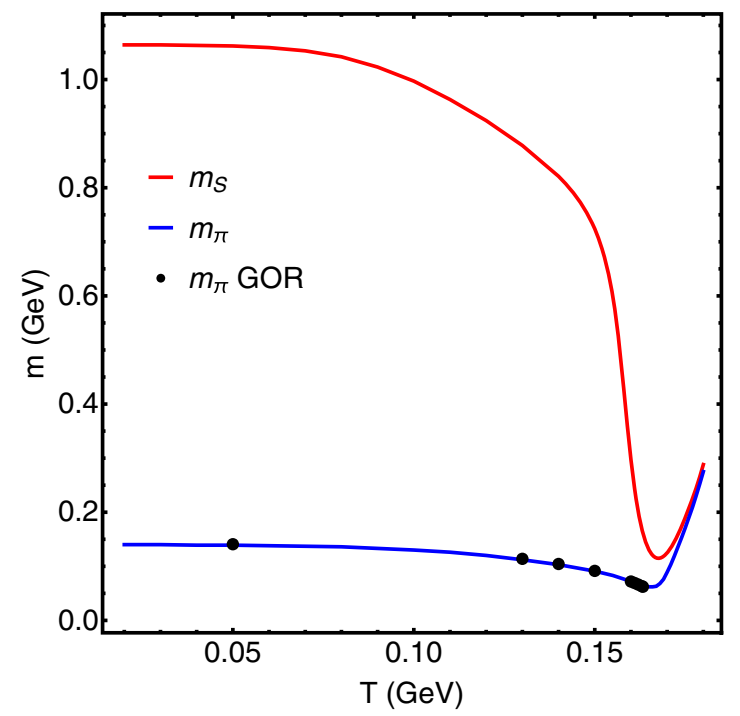

FIG. 4. Masses of the scalar meson and pion at finite temperature. The red and blue lines are extracted from the peaks of the spectral functions, representing results for the scalar meson and pion, respectively. The black dots are extracted via Eq. (51). different. From $T=0.166 \mathrm{GeV}$ to $T=0.175 \mathrm{GeV}$, we can see that the masses of both scalar and pseudoscalar mesons increase, together with the broadening of the widths. Such a behavior is consistent with the 4D effective model studies [12-14,17,18]. Physically, this is probably related to the transition from bound states to resonances, though the exact details from the holographic framework are still unclear and need further study in the future.

To be clearer, we extract the temperature dependence of the masses of the scalar meson $\left(m_{S}\right)$ and pion $\left(m_{\pi}\right)$ in Fig. 4. In the figure, the red and blue lines represent $m_{S}(T)$ and $m_{\pi}(T)$, respectively. From the figure, $m_{S}$ decreases rapidly below $T_{c p}$, from its vacuum value $m_{S}=1.06 \mathrm{GeV}$ to $m_{S}=$ $0.115 \mathrm{GeV}$ around $T_{c p}$, an almost $90 \%$ reduction. As for the pion, $m_{\pi}$ also decreases below $T_{c p}$, from its vacuum value of about $m_{\pi}=0.140 \mathrm{GeV}$ to $m_{\pi}=0.062 \mathrm{GeV}$ around $T_{c p}$, an almost $60 \%$ reduction. It is also quite interesting to see that qualitatively the results for the pion are consistent with Son and Stephanov's prediction [23,24] and the lattice simulations in Refs. [10,11]. Quantitatively, the holographic model gives almost double the reduction, with a rate of $60 \%$, while the reduction rates from Refs. [23,24] and Refs. [10,11] are around 30\% and $20 \%$, respectively. Since a reduction of the pion mass would enhance the low-momentum distribution of the pion, this effect contributes more in the relevant physics from our 
holographic prediction. Of course, the exact value of the enhancement depends on the fireball's evolution model, and this is outside of the scope of this work. Also, the holographic prediction is consistent with the NJL model prediction with gluon condensation [16], and it is in contrast to that in the NJL model without gluon condensation. In some sense, the gluon dynamics have been considered correctly, though in an implicit way. Finally, one can easily find that above $T_{c p}, m_{\pi}$ increases as well as the scalar meson, which is consistent with the 4D studies $[12-14,17,18]$. Moreover, the degeneracy of the scalar meson and pion is observed in the mass spectrum above $T_{c p}$, which reveals the restoration of the broken symmetry at the hadronic spectrum level. From another perspective, we find that the peaks for the spectral functions for the scalar and pseudoscalar modes above $T_{c}$, as well as those below $T_{c}$, are all determined by the quasinormal-mode frequencies $\omega_{0}$ in the complex $\omega$ plane. However, the relationship between quasinormal modes and spectral functions is complicated, and we will investigate these topics in a future article [118].

\section{E. The GOR relation at finite temperature}

In the previous section we extracted the masses from the spectral functions. Here, we will follow Ref. [38] and try to derive a different way to calculate the mass below $T_{c}$. Going back to Eq. (25), one can prove that it is equivalent to

$$
\begin{gathered}
\varphi^{\prime \prime}+\left(A^{\prime}-\Phi^{\prime}\right) \varphi^{\prime}-\frac{e^{2 A} g_{5}^{2} \chi^{2}}{f}(\varphi-\pi)=0, \\
\pi^{\prime}-\frac{\omega^{2} e^{-2 A} \varphi^{\prime}}{g_{5}^{2} f \chi^{2}}=0 .
\end{gathered}
$$

The main observation is that with $f\left(z_{h}\right)=0$ in Eq. (46b), the boundary condition in the IR could not be the normal real regular condition. The wave-like solution, i.e., the infalling and outgoing boundary conditions, appears. One has to calculate the spectral functions. Thus, we will make a naive assumption that the peak location for the pion below $T_{c}$ is not related to $f(z)$ in Eq. (46b), which only generates the widths at temperatures below $T_{c}$. Under such an assumption, one replaces $f(z)$ in Eq. (46b) with $f(z) \equiv$ 1 at zero temperature, and obtains

$$
\begin{gathered}
\varphi^{\prime \prime}+\left(A^{\prime}-\Phi^{\prime}\right) \varphi^{\prime}-\frac{e^{2 A} g_{5}^{2} \chi^{2}}{f}(\varphi-\pi)=0, \\
\pi^{\prime}-\frac{m_{\pi}^{2} e^{-2 A} \varphi^{\prime}}{g_{5}^{2} \chi^{2}}=0,
\end{gathered}
$$

where we have replaced $\omega^{2}$ with $m_{\pi}^{2}$ explicitly. Though only $\omega^{2}$ corresponding to the normalizable mode can be considered as pion mass.
Then, the process is similar to the derivation of the GOR relation at zero temperature done in Ref. [38]. When approaching the chiral limit $m_{q} \rightarrow 0, m_{\pi} \rightarrow 0$, one can construct the solution with a very small quark mass from the Goldstone mode in the chiral limit. Given the leading solution $\tilde{\varphi}$ and $\pi \equiv 0$, one can try to construct the next order for $\pi$ as

$$
\delta \pi(z)=\int_{0}^{z} d u \frac{m_{\pi}^{2} u^{3}}{\chi^{2}} \frac{\tilde{\varphi}^{\prime}}{g_{5}^{2} u} .
$$

It is easy to check that the above integration is divergent in the UV if $m_{q}$ equals zero exactly. However, if there is any finite $m_{q}$, the divergence would be removed. Thus, one can parametrize the divergence in $m_{q}$. Considering a small $m_{q}$ in $\chi$, the divergence in the chiral limit tells us that the contribution of the integration is mainly from the UV. Actually, with a very small $m_{q}$, the contribution is from a small $z$. Thus, one can get

$$
\delta \pi(z) \sim m_{\pi}^{2} f_{\pi, T}^{2} \int_{0}^{z} d u \frac{u^{3}}{\chi^{2}},
$$

where the temperature-dependent pion decay constant $f_{\pi, T}$ is defined as $f_{\pi, T}^{2}=\left.\frac{\tilde{\varphi}^{\prime}}{g_{5}^{2} z}\right|_{z \rightarrow 0}$. In the small- $m_{q}$ limit, the integration can be obtained as $1 /\left(2 m_{q} \sigma\right)$. Thus, we have

$$
\delta \pi(z) \sim \frac{m_{\pi}^{2} f_{\pi, T}^{2}}{2 m_{q} \sigma} .
$$

Considering the exact chiral limit, this solution should be the massless Goldstone mode with $\pi \equiv 1$, and we have the GOR relation at finite temperature,

$$
m_{\pi}^{2} f_{\pi, T}^{2}=2 m_{q} \sigma .
$$

For a small $m_{q}, f_{\pi, T}$ and $\sigma$ can be extracted from the solutions of $\tilde{\varphi}$ and $\chi$. Then, by using this relation, one can get $m_{\pi}$ at finite temperature.

Since the above derivation depends on the assumption given at the beginning of this section, we make a numerical check of this relation. The black dots in Fig. 4 are obtained from the finite- $T$ GOR relations in Eq. (51). In Fig. 4, we can see that it agrees very well with the blue line from the spectral functions. This might be considered as a numerical check of our assumption. It might provide a simpler way to extract the pole mass at temperatures below $T$. Of course, when the temperature is above $T_{c}$, the Goldstone mode in the chiral limit disappears, and the GOR relation derived here cannot be used. From the discussion in this section, we can see that the coupling with $\varphi$ is quite important to realize the Goldstone nature of the pion. The scenario neglecting such couplings in Refs. [73,74] might not be a good 
approximation at low temperature, especially in the chiral limit.

\section{PION QUASIPARTICLES AT FINITE ISOSPIN DENSITY}

In the previous section we presented a careful analysis of scalar-type quasiparticles only at finite temperatures. However, in heavy-ion collisions, the nuclear matter density might also be very important at certain collision energies. The recent experimental project BES [90-92] was designed mainly to probe the baryon number density effect, and in particular to determine the CEP in the $T-\mu_{B}$ plane. Besides the baryon number, the isospin number is another conserved charge in QCD, and its density might also change the properties of the medium. For example, a finite isospin density might be generated by the different numbers of protons and neutrons in the initial nuclei. This might lead to an imbalance between charged pions in the final distribution [119]. Also, the pion superfluid phase, consisting of condensed charged pions, might have been produced in experiments [5-7]. Thus, investigating the isospin density $n_{I}$ (or the isospin chemical potential $\mu_{I}$ ) effect has attracted growing attention.

At finite $T$ and $\mu_{B}$, one of the main interests is the chiral phase transition, which is related to the symmetry breaking from $S U(2)_{V} \times S U(2)_{A} \quad$ [or, equivalently, $S U(2)_{L} \times$ $S U(2)_{R}$ ] to $S U(2)_{V}$. As shown in previous sections, in this transition the neutral pion $\pi^{0}$ together with the two charged pions $\pi^{+}$and $\pi^{-}$play the role of Goldstone bosons in the chiral limit and pseudo-Goldstone bosons with finite quark mass. Son and Stephanov [120] suggested that with a sufficiently large $\mu_{I}$, the nuclear matter might transit from the normal phase to the pion superfluid phase. This is supported by both lattice simulations [121] and model studies [17,18]. In this transition, the $S U(2)_{V}$ symmetry would be broken to $U_{I}(1)$, which is the Abelian subgroup of $S U(2)_{V}$. Since the isospin numbers $I_{3}$ of $\pi^{+}, \pi^{0}$, and $\pi^{-}$are different, the three pions might split at finite $\mu_{I}$. One of the charged pions would become the Goldstone boson of this symmetry-breaking process. Since one of the main goals of this work is to investigate the relationship between the hadron spectrum and phase transitions, in this section we focus on the isospin density effect, which provides a possibility to probe another kind of phase transition.

In the holographic framework, the isospin density effect has been studied in the hard-wall model in Refs. [93-97]. It has been shown that above $\mu_{I}$ charged pions tend to form a Bose-Einstein condensate. In our previous work [99], we extended this study to both finite temperature and isospin density, and we obtained the phase diagram in the $T-\mu_{I}$ plane, as shown in Fig. 5. In the figure, a $\Lambda$ type of phase boundary (the red solid line) is observed. Below the phase boundary, i.e., at relatively large $\mu_{I}$ and low $T$, the pion condensed phase forms. Outside this region, the nuclear

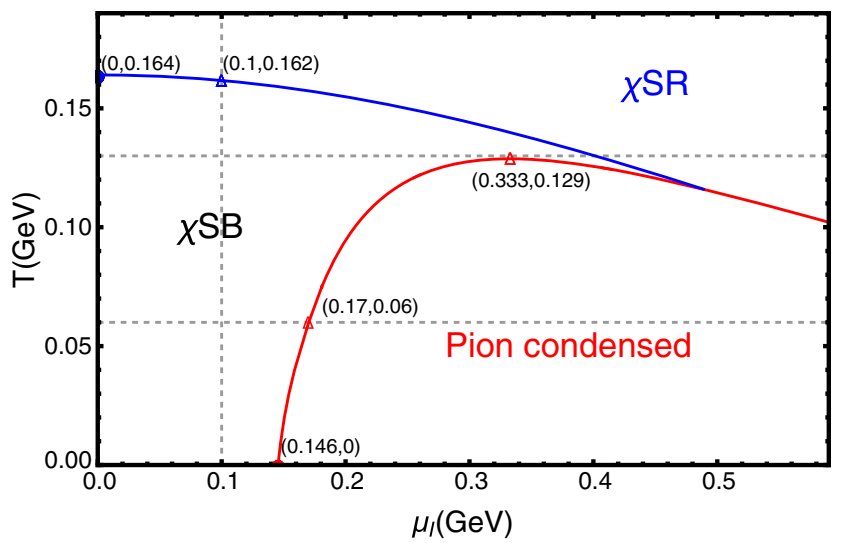

FIG. 5. QCD phase diagram at finite temperature and finite isospin chemical potential [99]. The blue line is the phase boundary between the normal chiral-symmetry-broken $(\chi \mathrm{SB})$ phase and chiral-symmetry-restored $(\chi \mathrm{SR})$ phase. The pion condensed phase is bounded by the red line and the $\mu_{I}$ axis. The maximal temperature of pion condensation is $T_{c, t o p}=129 \mathrm{MeV}$, labeled at the peak of the red line. The masses of pions along the three grey lines are studied in Sec. III B.

matter is in the normal phase. The chiral transition line is also shown by the blue line.

\section{A. Spectral functions of pseudoscalar modes}

According to the holographic recipe, the conserved current is dual to the gauge field in Eq. (3). The isospin current $\bar{q} \gamma_{\mu} t^{3} q$ is dual to $V_{\mu}^{3}$. At finite isospin density, one has to consider a nonzero $V_{\mu}^{3}$. Generally, the solution should be solved using a certain kind of gravity system coupled with the soft-wall AdS/QCD model action. But it is difficult to solve the action with the full backreaction. Thus, for simplicity, in the sense of theprobe limit, we take the AdS-Reissner-Nordström metric solution of the action, which couples the $F_{L}^{2}+F_{R}^{2}$ terms in Eq. (1) with the 5D Einstein-Hilbert action. Thus, the metric in Eqs. (6) and (7) would be replaced with

$$
\begin{gathered}
d s^{2}=e^{2 A(z)}\left(f(z) d t^{2}-d x^{i} d x_{i}-\frac{1}{f(z)} d z^{2}\right), \\
f(z)=1-\left(1+\mu_{I}^{2} z_{h}^{2}\right) \frac{z^{4}}{z_{h}^{4}}+\mu_{I}^{2} \frac{z^{6}}{z_{h}^{4}}
\end{gathered}
$$

together with a nonzero $V_{0}^{3}$ of the following form:

$$
V_{0}^{3}(z)=\mu_{I}\left(1-\frac{z^{2}}{z_{h}^{2}}\right)
$$

Here, $\mu_{I}$ is the isospin density and $z_{h}$ is the horizon where $f(z)=0$. In this case, the temperature is defined by the surface gravity as 


$$
T=\left|\frac{f^{\prime}\left(z_{h}\right)}{4 \pi}\right|=\frac{2-\mu_{I}^{2} z_{h}^{2}}{2 \pi z_{h}}
$$

Since we focus on the pion superfluid transition, in which the pseudoscalar mode is relevant, we will only consider pions in this section. At finite temperature, the three pseudoscalar modes $\pi^{1}, \pi^{2}$, and $\pi^{3}$ in Eq. (17) are symmetric under rotation in isospin space. However, with finite isospin chemical potential $\mu_{I}$, such symmetry is broken, and we expect the splits of these three modes. This can be easily read from the effective action

$$
\begin{aligned}
S_{P S}= & -\frac{1}{2 g_{5}^{2}} \int d^{5} x \sqrt{-g} e^{-\Phi}\left[\sum _ { i = 1 } ^ { 3 } \left\{g^{\mu \nu} g^{z z} \partial_{z} \partial_{\mu} \varphi^{i} \partial_{z} \partial_{\nu} \varphi^{i}-g_{5}^{2} \chi^{2}\left[g^{\mu \nu} \partial_{\mu} \varphi^{i} \partial_{\nu} \varphi^{i}\right.\right.\right. \\
& \left.\left.+g^{\mu \nu} \partial_{\mu} \pi^{i} \partial_{\nu} \pi^{i}+g^{z z}\left(\partial_{z} \pi^{i}\right)^{2}\right]\right\}-g_{5}^{2} \chi^{2}\left\{g^{00}\left(V_{0}^{3}\right)^{2}\left[\left(\pi^{1}\right)^{2}+\left(\pi^{2}\right)^{2}\right]\right. \\
& \left.\left.+2 g^{00} V_{0}^{3}\left(\pi^{1} \partial_{0} \pi^{2}-\pi^{2} \partial_{0} \pi^{1}\right)-2 g^{00} V_{0}^{3}\left(\pi^{1} \partial_{0} \varphi^{2}-\pi^{2} \partial_{0} \varphi^{1}\right)-2 g^{\mu \nu} \sum_{i=1}^{3} \partial_{\mu} \varphi^{i} \partial_{\nu} \pi^{i}\right\}\right] .
\end{aligned}
$$

For later convenience, we redefine $\pi^{1}$ and $\pi^{2}$ as

$$
\pi^{ \pm}=\frac{1}{\sqrt{2}}\left(\pi^{1} \mp i \pi^{2}\right), \quad \varphi^{ \pm}=\frac{1}{\sqrt{2}}\left(\varphi^{1} \mp i \varphi^{2}\right),
$$

where $\pi^{ \pm}$and $\varphi^{ \pm}$represent the degrees of freedom for charged pions. We denote the modes $\pi^{3}, \varphi^{3}$ as $\pi^{0}, \varphi^{0}$ to specify the charge difference.

Like the case at finite temperature, we only consider the time $t$ and $z$ dependence of the three modes and transform all of them to frequency space as

$$
\begin{gathered}
\pi^{0}(t, z)=\frac{1}{2 \pi} \int d \omega_{0} e^{-i \omega_{0} t} \pi^{0}\left(\omega_{0}, z\right), \\
\pi^{ \pm}(t, z)=\frac{1}{2 \pi} \int d \omega_{ \pm} e^{-i \omega_{ \pm} t} \pi^{ \pm}\left(\omega_{ \pm}, z\right), \\
\varphi^{0}(t, z)=\frac{1}{2 \pi} \int d \omega_{0} e^{-i \omega_{0} t} \varphi^{0}\left(\omega_{0}, z\right), \\
\varphi^{ \pm}(t, z)=\frac{1}{2 \pi} \int d \omega_{ \pm} e^{-i \omega_{ \pm} t} \varphi^{ \pm}\left(\omega_{ \pm}, z\right) .
\end{gathered}
$$

Then, one can obtain the equations of motion for the three modes as

$$
\begin{gathered}
\varphi^{0^{\prime \prime}}+\left(A^{\prime}-\Phi^{\prime}\right) \varphi^{0^{\prime}}-\frac{e^{2 A} g_{5}^{2} \chi^{2}}{f}\left(\varphi^{0}-\pi^{0}\right)=0, \\
\pi^{0^{\prime \prime}}+\left(3 A^{\prime}+\frac{f^{\prime}}{f}-\Phi^{\prime}+\frac{2 \chi^{\prime}}{\chi}\right) \pi^{0^{\prime}}-\frac{\left(\varphi^{0}-\pi^{0}\right) \omega^{2}}{f^{2}}=0,
\end{gathered}
$$

and

$$
\begin{gathered}
\varphi^{ \pm^{\prime \prime}}+\left(A^{\prime}-\Phi^{\prime}\right) \varphi^{ \pm^{\prime}} \\
-\frac{e^{2 A} g_{5}^{2} \chi^{2}}{\omega_{ \pm} f}\left[\omega_{ \pm} \varphi^{ \pm}-\left(\omega_{ \pm} \pm V_{0}^{3}\right) \pi^{ \pm}\right]=0 \\
\pi^{ \pm^{\prime \prime}}+\left(3 A^{\prime}+\frac{f^{\prime}}{f}-\Phi^{\prime}+\frac{2 \chi^{\prime}}{\chi}\right) \pi^{ \pm^{\prime}} \\
-\frac{\left(\omega_{ \pm} \pm V_{0}^{3}\right)\left[\omega_{ \pm} \varphi^{ \pm}-\left(\omega_{ \pm} \pm V_{0}^{3}\right) \pi^{ \pm}\right]}{f^{2}}=0 .
\end{gathered}
$$

Also, it is not difficult to derive the on-shell action for the neutral pion as

$$
\begin{aligned}
S_{\pi 0}^{\mathrm{on}}= & -\frac{1}{2 g_{5}^{2}} \int d \omega e^{A-\Phi}\left[e^{2 A} g_{5}^{2} f \chi^{2} \pi^{0}(-\omega, z) \pi^{0^{\prime}}(\omega, z)\right. \\
& \left.-\omega^{2} \varphi^{0}(-\omega, z) \varphi^{0^{\prime}}(\omega, z)\right]\left.\right|_{z=z_{h}} ^{z=z_{h}} .
\end{aligned}
$$

The charged pions' on-shell actions are

$$
\begin{aligned}
S_{\pi \pm}^{\text {on }}= & -\frac{1}{2 g_{5}^{2}} \int d \omega e^{A-\Phi}\left[e^{2 A} g_{5}^{2} f \chi^{2} \pi^{ \pm}(-\omega, z) \pi^{\mp^{\prime}}(\omega, z)\right. \\
& \left.-\omega^{2} \varphi^{ \pm}(-\omega, z) \varphi^{\mp^{\prime}}(\omega, z)\right]\left.\right|_{z=\epsilon} ^{z=z_{h}} .
\end{aligned}
$$

To get the on-shell action, one has to solve the equations of motion. Before that, the UV and IR boundary conditions should be specified. At the UV boundary, the asymptotic expansion can be derived as

$$
\begin{aligned}
& \varphi^{0}(z \rightarrow 0)=c_{f}+\varphi_{2} z^{2}-\frac{1}{2} \zeta^{2} g_{5}^{2} m^{2} \pi_{0} z^{2} \log (z)+\mathcal{O}\left(z^{3}\right), \\
& \pi^{0}(z \rightarrow 0)=\pi_{0}+c_{f}+\pi_{2} z^{2}-\frac{1}{2} \pi_{0} \omega^{2} z^{2} \log (z)+\mathcal{O}\left(z^{3}\right),
\end{aligned}
$$


and

$$
\begin{aligned}
\varphi^{ \pm}(\omega, z \rightarrow 0)= & \frac{g_{5}^{2} m^{2} \zeta^{2}\left[\omega \varphi_{0}^{ \pm}-\left(\omega \pm \mu_{I}\right) \pi_{0}^{ \pm}\right] z^{2} \log (z)}{2 \omega} \\
& +\varphi_{0}^{ \pm}+\varphi_{2}^{ \pm} z^{2}+\mathcal{O}\left(z^{3}\right), \\
\pi^{ \pm}(\omega, z \rightarrow 0)= & \frac{1}{2}(\omega \pm \mu)\left[\varphi_{0}^{ \pm} \omega-\left(\omega \pm \mu_{I}\right) \pi_{0}^{ \pm}\right] z^{2} \log (z) \\
& +\pi_{0}^{ \pm}+\pi_{2}^{ \pm} z^{2}+\mathcal{O}\left(z^{3}\right),
\end{aligned}
$$

for $\pi^{0}, \varphi^{0}$ and $\pi^{ \pm}, \varphi^{ \pm}$respectively, where $\pi_{0}, c_{f}, \pi_{2}, \varphi_{2}, \varphi_{0}^{ \pm}$, $\varphi_{2}^{ \pm}, \pi_{0}^{ \pm}$, and $\pi_{2}^{ \pm}$are integral constants.

To get the retarded Green functions, the infalling condition should be imposed at the IR boundary. Thus, the expansions at the IR boundary are

$$
\begin{aligned}
\varphi^{0}\left(z \rightarrow z_{h}\right)= & \left(z_{h}-z\right)^{\frac{i \omega z_{h}}{2 \mu_{I}^{2} z_{h}^{2}-4}}\left\{\frac{2 i c_{0}^{2} g_{5}^{2} \pi_{\mathrm{h} 0}\left(\mu_{I}^{2} z_{h}^{2}-2\right)\left(z-z_{h}\right)}{\omega z_{h}^{2}\left(2 \mu_{I}^{2} z_{h}^{2}+i \omega z_{h}-4\right)}\right. \\
& \left.+\mathcal{O}\left[\left(z-z_{h}\right)^{2}\right]\right\}+c_{h 0}, \\
\pi^{0}\left(z \rightarrow z_{h}\right)= & \left(z_{h}-z\right)^{\frac{i \omega z_{h}}{2 \mu_{I}^{2} z_{h}^{2}-4}}\left\{\pi_{h 0}+\mathcal{O}\left(z-z_{h}\right)\right\}+c_{h 0},
\end{aligned}
$$

and

$$
\begin{aligned}
\varphi^{ \pm}\left(z \rightarrow z_{h}\right)= & \left(z_{h}-z\right)^{\frac{i \omega z_{h}}{2 \mu_{I}^{2} z_{h}^{2}-4}}\left\{\frac{2 i c_{0}^{2} g_{5}^{2}\left(z-z_{h}\right)\left(\mu_{I}^{2} z_{h}^{2}-2\right) \pi_{\mathrm{h} 0}^{ \pm}}{\omega z_{h}^{2}\left(2 \mu_{I}^{2} z_{h}^{2}+i \omega z_{h}-4\right)}\right. \\
& \left.+\mathcal{O}\left[\left(z-z_{h}\right)^{2}\right]\right\}+c_{h 0}^{ \pm}, \\
\pi^{ \pm}\left(z \rightarrow z_{h}\right)= & \left(z_{h}-z\right)^{\frac{i z_{h}}{2 \mu_{I}^{2} z_{h}^{2}-4}}\left\{\pi_{\mathrm{h} 0}^{ \pm}+\mathcal{O}\left(z-z_{h}\right)\right\}+c_{h 0}^{ \pm},
\end{aligned}
$$

where $\pi_{h 0}, c_{h 0}, \pi_{h 0}^{ \pm}$, and $c_{h 0}^{ \pm}$are integral constants. Under these conditions, one can solve all of the integral constants and get the spectral function through the following expressions ${ }^{7}$ :

$$
\begin{aligned}
\rho\left(\pi^{0}, \omega\right) & =-\frac{1}{\pi} \operatorname{Im} G_{\pi^{0}}^{R}(\omega) \\
& =\operatorname{Im}\left[\frac{1}{4 \pi \pi_{0}} m_{q}^{2} \zeta^{2}\left(4 \pi_{2}-\pi_{0} \omega^{2}\right)\right],
\end{aligned}
$$

\footnotetext{
${ }^{7}$ The parameter $c_{f}$ and $\varphi_{0}^{ \pm}$are set to zero. Certain real terms inside the imaginary function are neglected.
}

and

$$
\begin{aligned}
\rho\left(\pi^{ \pm}, \omega\right) & =-\frac{1}{\pi} \operatorname{Im} G_{\pi^{ \pm}}^{R}(\omega) \\
& =\operatorname{Im}\left\{\frac{1}{4 \pi \pi_{0}^{ \pm}} m_{q}^{2} \zeta^{2}\left[4 \pi_{2}^{ \pm}-(\omega \pm \mu)^{2} \pi_{0}^{ \pm}\right]\right\} .
\end{aligned}
$$

\section{B. Mass spectra of pion quasiparticles and the Goldstone boson}

The pion superfluid transition is connected with the breaking of $S U(2)_{V}$ symmetry, which is an exact symmetry in both the chiral limit and cases with finite quark masses. So, in this section we only consider the realistic case with a physical quark mass. Taking $m_{q}=3.22 \mathrm{MeV}$ and solving the equations of motion (EOMs) in Eqs. (58) and (59), one can obtain the spectral functions.

First, we fix $\mu_{I}=0.1 \mathrm{GeV}$ and investigate the temperature-dependent behavior of the three modes, i.e., along the vertical gray line in Fig. 5. The spectral functions for $\pi^{+}, \pi^{0}$, and $\pi^{-}$are shown in Figs. 6(a), 6(b), and 6(c), respectively, for temperatures $T=0.07,0.13$, and $0.165 \mathrm{GeV}$. From the locations of the peaks, we can see that at low temperature and finite isospin chemical potential the masses of $\pi^{+}, \pi^{0}$, and $\pi^{-}$are split. For example, at $T=0.07 \mathrm{GeV}, m_{\pi^{+}} \approx 0.057 \mathrm{GeV}$ and $m_{\pi^{0}} \approx 0.136 \mathrm{GeV}$, which are smaller than their vacuum values, while $m_{\pi^{-}} \approx 0.210 \mathrm{GeV}$, which is larger than its vacuum value. Moreover, from the locations of the peaks one can see that, with increasing temperature, the masses of $\pi^{-}$and $\pi^{0}$ decrease monotonically, while $m_{\pi^{+}}$increases at low temperature. This result might be reasonable. Considering that the pion condensed phase appears at $T_{c, \pi}=0, \mu_{I} \approx 0.146 \mathrm{GeV}$, a possible coherent fraction of $\pi^{+}$might form at $\mu_{I}=0.1 \mathrm{GeV}$, which would reduce the energy needed to excite the $\pi^{+}$. The increasing temperature tends to destroy the coherence of particles. Thus, it would have a contrasting effect on $m_{\pi^{+}}$.

For temperatures above the blue line in Fig. 5, we take $T=0.166,0.170$, and $0.175 \mathrm{GeV}$ as examples and show the results in Fig. 6(d). From the figure, we can see that although the heights of the peaks are different for the three modes, the locations of the peaks are almost the same. This shows that the high-temperature modes are still governed by the chiral phase transition.

To be clearer, we extract the temperature-dependent masses and plot them in Fig. 7. The decreasing of $m_{\pi^{-}}$ and $m_{\pi^{0}}$ can be clearly seen from the black and blue lines, respectively. The result for $m_{\pi^{0}}$ is almost the same as that at $\mu_{I}=0$, since the third component of isospin number $\left(I_{3}\right)$ of $\pi^{0}$ is zero. For $m_{\pi^{-}}$, which is enhanced by $\mu_{I}$, near $T=0$ it increases to about $0.235 \mathrm{GeV}$. With increasing temperature, it decreases to $0.061 \mathrm{GeV}$ near the pseudotransition temperature $T_{c p}=0.162 \mathrm{GeV}$. For $m_{\pi^{+}}$, a little bump appears near the chiral crossover point. At first glance, 

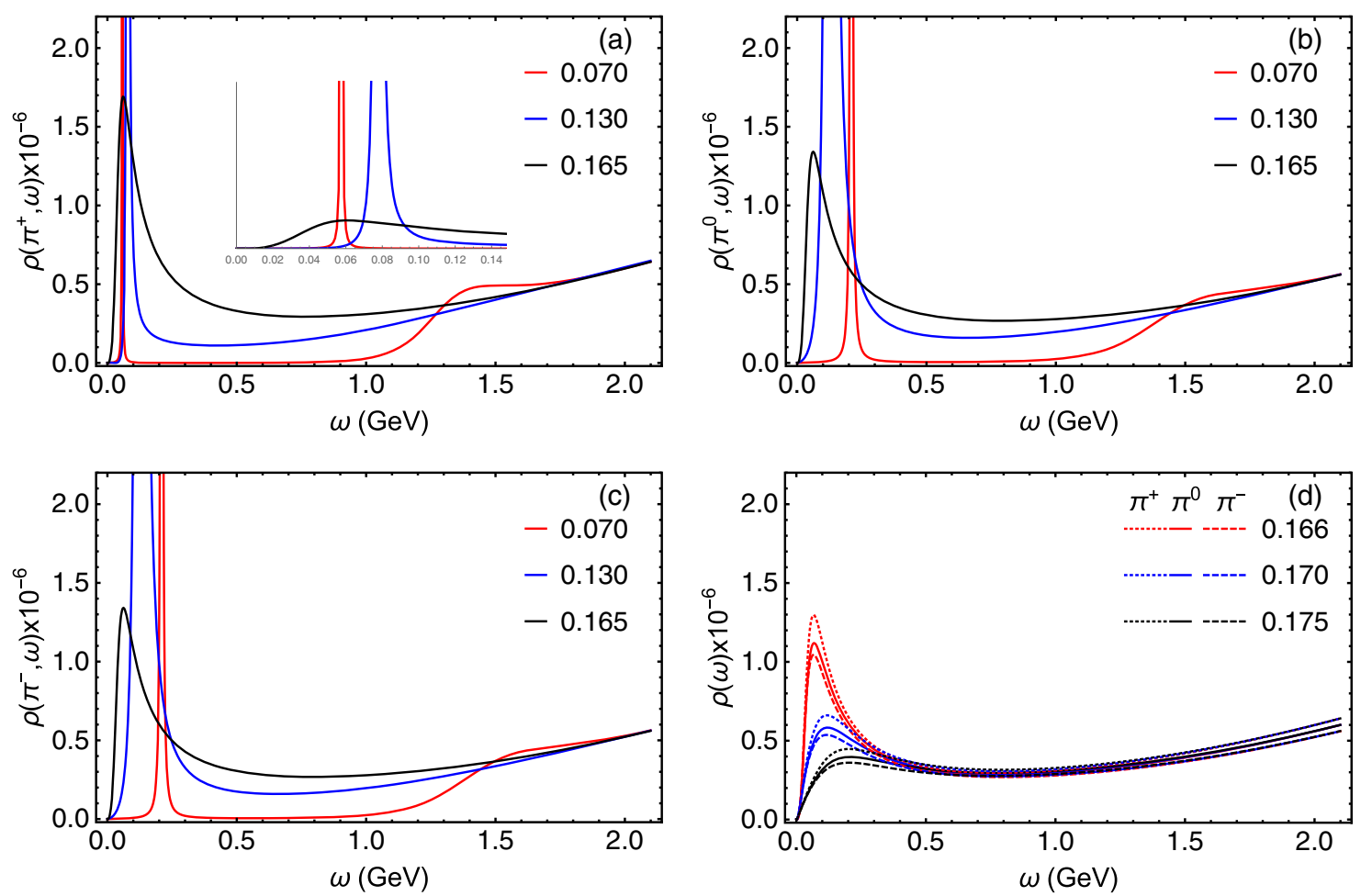

FIG. 6. Spectral functions of (a) $\rho\left(\pi^{+}, \omega\right)$ [an enlarged view for frequency in the interval $(0,0.15)$ is shown], (b) $\rho\left(\pi^{0}, \omega\right)$, and (c) $\rho\left(\pi^{-}, \omega\right)$, at $\mu_{I}=0.1 \mathrm{GeV}$. The red, blue, and black solid lines represent results below $T_{c p}$, at $T=0.07,0.13$, and $0.165 \mathrm{GeV}$, respectively. (d) Spectra functions at $\mu_{I}=0.1 \mathrm{GeV}$ and $T$ above $T_{c p}$, with the dotted line for $\pi^{+}$, solid line for $\pi^{0}$, and dashed line for $\pi^{-}$. The red, blue, and black lines represent the results for $T=0.166,0.170$, and $0.175 \mathrm{GeV}$, respectively.

this is quite strange. Actually, it could also be reasonable. Here, the increasing temperature has two effects. One is to destroy the coherent fraction of $\pi^{+}$, while the other one is to decrease the chiral condensate. The former effect would lead to an increase of $m_{\pi^{+}}$while the latter leads to a decrease, as discussed in Sec. II. Therefore, the bump is the result of the competition between these two effects. It is obvious that the three pions become degenerate at temperatures above $T_{c p}$, which is mainly controlled by the chiral phase transition.

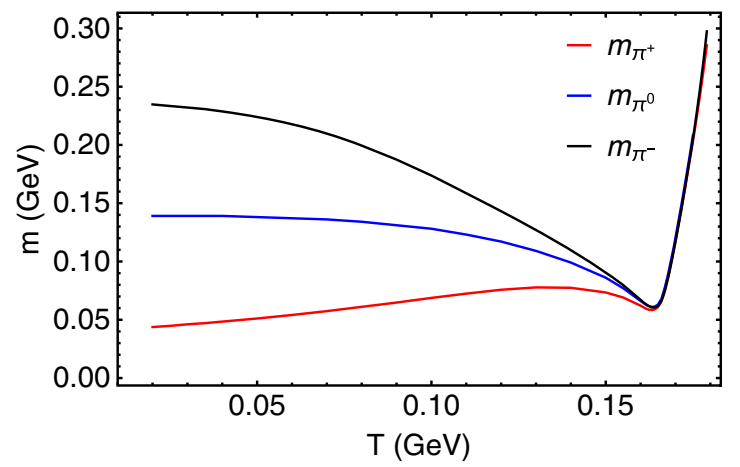

FIG. 7. Temperature-dependent behaviors of pions masses at $\mu_{I}=0.1 \mathrm{GeV}$. The black, blue, and red lines are for $m_{\pi^{-}}, m_{\pi^{0}}$, and $m_{\pi^{+}}$, respectively.
Now we turn to the $\mu_{I}$ dependence of the three modes. We fix $T=0.06 \mathrm{GeV}$ and vary $\mu_{I}$, i.e., along the lower gray horizontal line in Fig. 5. The spectral functions of $\pi^{+}, \pi^{0}$, and $\pi^{-}$are presented in Figs. 8(a), 8(b), and 8(c), respectively. From Fig. 8(b), again, we find that the dependence of $m_{\pi^{0}}$ on $\mu_{I}$ is rather weak at low temperature. The locations of the peaks move slightly towards $\omega=0$. In Figs. 8(a) and 8(c), the $\mu_{I}$ dependence of $m_{\pi^{+}}$is opposite that of $m_{\pi^{-}}$. The former decreases with $\mu_{I}$ while the latter increases. In particular, the most interesting thing is the appearance of a massless $\pi^{+}$at $\mu_{I} \approx 0.170 \mathrm{GeV}$. Another observation is that the widths of the peaks increase with $\mu_{I}$ at such a low temperature.

We also display the $\mu_{I}$ dependence of the quasiparticles' masses in Fig. 9. Qualitatively, the picture of the mass splitting is in agreement with the previous study in the hard-wall model $[94,96,97]$. At $T=0.06 \mathrm{GeV}, \mu_{I}=0$, the masses of the three pions are degenerate at $m \approx 0.138 \mathrm{GeV}$. When $\mu_{I}$ increases to $0.170 \mathrm{GeV}$, the $\pi^{+}$becomes massless. This is related to a certain instability. In fact, $T=$ $0.06 \mathrm{GeV}, \mu_{I}=0.17 \mathrm{GeV}$ is located exactly at the phase boundary between the pion condensed phase and normal phase. Above $\mu_{I}=0.17 \mathrm{GeV}$, the $S U(2)_{V}$ symmetry is broken to its subgroup $U_{I}(1)$, and $\pi^{+}$becomes the massless Goldstone boson of the symmetry breaking. Interestingly, we observe the realization of the Goldstone theorem in the 

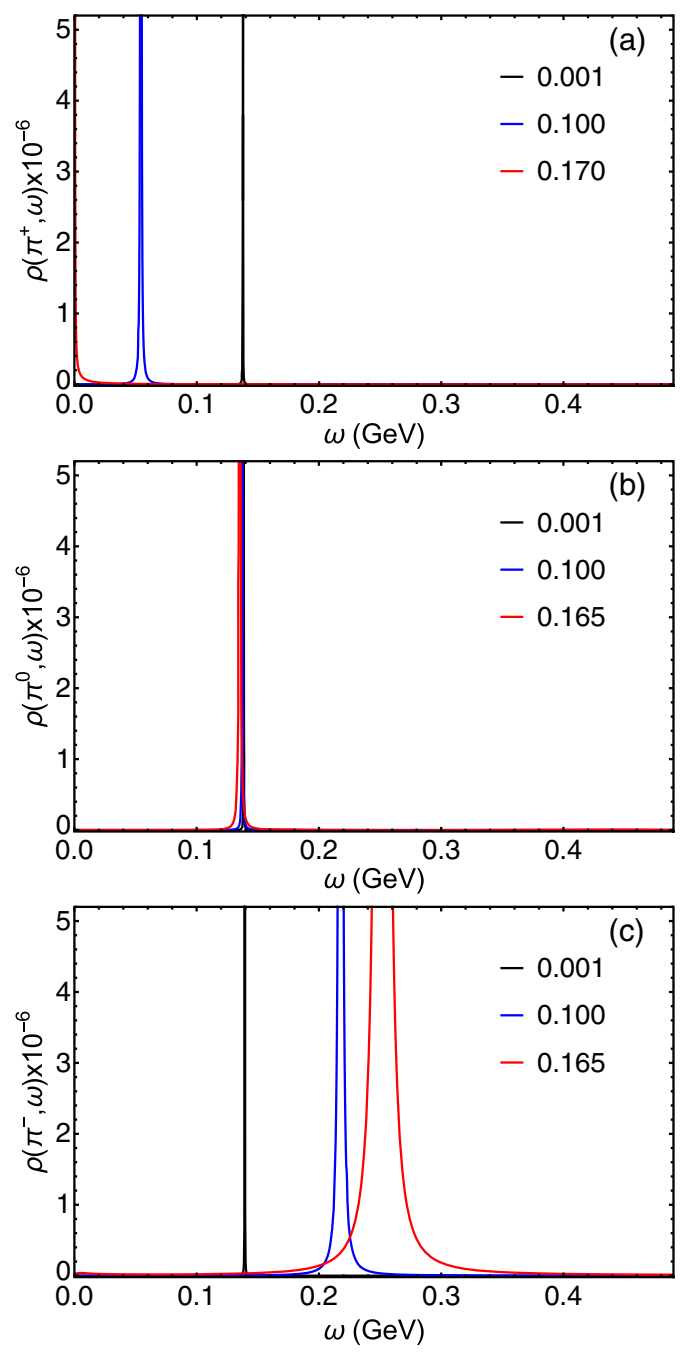

FIG. 8. Spectral functions for (a) $\rho\left(\pi^{+}, \omega\right)$, (b) $\rho\left(\pi^{0}, \omega\right)$, and (c) $\rho\left(\pi^{-}, \omega\right)$, at $T=0.06 \mathrm{GeV}$. The black and blue lines represent results at $\mu_{I}=0.001$ and 0.100 , respectively. The red line represents results at $\mu_{I}=0.170 \mathrm{GeV}$ in panel (a) and $\mu_{I}=0.165 \mathrm{GeV}$ in panels (b) and (c).



FIG. 9. $\mu_{I}$ dependence of the pions' masses at $T=0.06 \mathrm{GeV}$. The black, blue, and red lines represent results for $m_{\pi^{-}}, m_{\pi^{0}}$, and $m_{\pi^{+}}$, respectively. $m_{\pi^{+}}$vanishes at $0.170 \mathrm{GeV}$, as shown by the black dot.

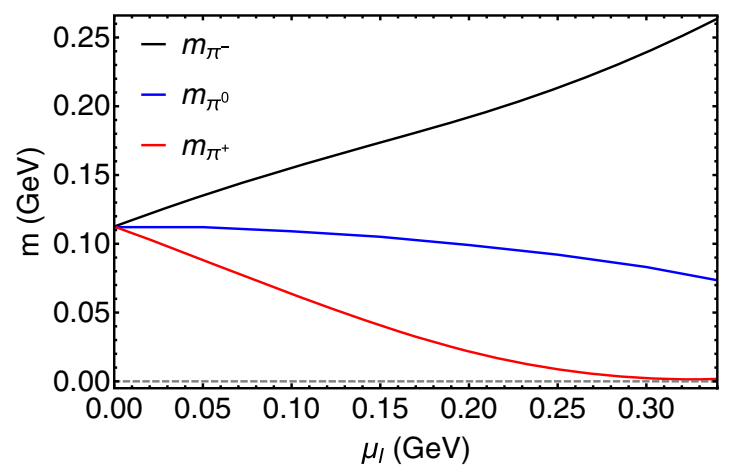

FIG. 10. $\mu_{I}$ dependence of the pions' masses at $T=0.13 \mathrm{GeV}$. The black, blue, and red lines represent results for $m_{\pi^{-}}, m_{\pi^{0}}$, and $m_{\pi^{+}}$, respectively.

holographic approach at both finite temperature and isospin density. As for $\pi^{-}$, its mass increases from $0.138 \mathrm{GeV}$ to around $0.253 \mathrm{GeV}$. The increasing mass split of $\pi^{+}$and $\pi^{-}$ might affect the distribution of the final charged pions if the fireball passes such an intermediate state.

Finally, as shown in Fig. 5, when the temperature is higher than $T_{c, t o p}=0.129 \mathrm{GeV}$, the condensed phase is destroyed, even with a very large $\mu_{I}$. Thus, we will also investigate the behavior of the mass spectral at high temperatures. We take $T=0.13 \mathrm{GeV}$ as an example. From our calculation, with larger widths, the peaks in the spectral functions are similar to those at $T=0.06 \mathrm{GeV}$, so we give the $\mu_{I}$ dependence of pion masses only. This is shown in Fig. 10. Qualitatively, the results for $\pi^{0}$ and $\pi^{-}$are similar to those at $T=0.06 \mathrm{GeV}$. As for $\pi^{+}$, the massless mode disappears even at very large $\mu_{I}$, which is consistent with the absence of pion superfluid at high temperatures.

\section{CONCLUSION}

In this work, we investigated the masses of light (pseudo) scalar mesons at finite temperature $T$ and isospin chemical potential $\mu_{I}$ (extracted from the spectral functions), as well as their relationship with the chiral phase transition and pion superfluid transition in a two-flavor soft-wall AdS/ QCD model.

In the chiral limit, charged and neutral pions were proved numerically and analytically to be massless Goldstone modes for any temperature below the critical temperature $T_{c}$ of the chiral phase transition. The mass of the scalar meson decreases from its vacuum value $1.05 \mathrm{GeV}$ to zero at $T_{c}=0.163 \mathrm{GeV}$. Above $T_{c}$, the masses of scalar and pseudoscalar mesons become degenerate and increase with temperature. This can be considered as the realization of chiral symmetry restoration at the hadronic level, which is consistent with the signal from the chiral condensate $\langle\bar{q} q\rangle$. Qualitatively, this picture is in good agreement with the Goldstone theorem [75,76] and expectations from theoretical analysis. In this sense, the theoretical consistency of the soft-wall model has been checked. 
To be more realistic, we studied physical quark mass cases, in which the chiral phase transition is a crossover. Qualitatively, the scalar masses with respect to temperature are similar to the chiral limit case except for the nonzero minimum at the pseudocritical temperature at $T_{c p}=$ $0.164 \mathrm{GeV}$. However, the main difference comes from pions. Below $T_{c p}$, their masses decrease from the vacuum value $0.140 \mathrm{GeV}$ to $0.062 \mathrm{GeV}$ at $T_{c p}$. This result agrees well with the general analysis by Son and Stephanov in Refs. [23,24], as well as lattice simulations in Refs. [10,11]. The reduction rate from our holographic study is about $60 \%$, which is larger than the $30 \%$ from Refs. [23,24] and $20 \%$ from Refs. [10,11]. This behavior might lead to a larger contribution from the mass decrease of pions to the enhancement of the lowmomentum distribution of pions in heavy-ion collisions. For temperatures above $T_{c p}$, the degeneration of scalar and pseudoscalar modes is observed again, and both the spectral increase with temperature, which is consistent with results from 4D models [12-14,17,18]. Phenomenologically, it might be a possible candidate for the signal of phase transitions in experiments.

Considering the growing interest in the effect of isospin density, we extended the above study to the situation with a finite isospin chemical potential $\mu_{I}$ and investigated the pion mass behaviors with finite $\mu_{I}$ and $T$. With a fixed $\mu_{I}$, e.g., $\mu_{I}=0.1 \mathrm{GeV}$, there are mass splits of $\pi^{+}, \pi^{0}$, and $\pi^{-}$ as a result of a finite $\mu_{I}$ at low temperature. The masses of $\pi^{+}$and $\pi^{-}$are shifted down and up, respectively, while $m_{\pi^{0}}$ is kept unchanged. This result is consistent with previous holographic studies at $T=0, \mu_{I} \neq 0[94,96,97]$. Phenomenologically, the mass splits of $\pi^{ \pm}$would affect the distribution of $\pi^{ \pm}$and contribute to the ratio of the multiplicity of charged pions $\left(\pi^{-}\right.$to $\left.\pi^{+}\right)$detected in experiments. Below $T_{c p}$, with the increasing temperature, $m_{\pi^{-}}$and $m_{\pi^{0}}$ decrease. $m_{\pi^{+}}$increases to a maximum value first, and then decreases with temperature. Above $T_{c p}$, the three modes become degenerate and increase with temperature, as shown in Fig. 7. With a fixed low temperature, e.g., $T=0.06 \mathrm{GeV}, m_{\pi^{+}}\left(m_{\pi^{-}}\right)$decreases (increases) with $\mu_{I}$, while $m_{\pi^{0}}$ depends weakly on $\mu_{I}$. The mass splits of pions increase rapidly with $\mu_{I}$. When $m_{\pi^{+}}=0$ and $\mu_{I}=0.17 \mathrm{GeV}$, an instability occurs and the pion condensed phase appears. There is a spontaneous symmetry breaking from $S U(2)_{V}$ to $U_{I}(1)$, and $\pi^{+}$is the Goldstone mode. With a fixed high temperature, e.g., $T=0.13 \mathrm{GeV}$, qualitatively the $\mu_{I}$ dependence of pions is similar to that at $T=0.06 \mathrm{GeV}$ with a relatively small $\mu_{I}$. However, one can no longer find the massless Goldstone mode at large $\mu_{I}$. This is consistent with our previous study [99] where we determined that no condensed phase exists above $T_{c, t o p}=0.129 \mathrm{GeV}$.

The current study checked the theoretical consistency of the soft-wall holographic framework. The qualitative behavior of the chiral phase transition and pion superfluidity transition can be well realized from both the order parameters and hadronic spectra. Furthermore, the $T$ and $\mu_{I}$ dependences of light scalar and pseudoscalar mesons might have interesting consequences in heavy-ion collisions. The LHC data indicate that the chemical freeze-out temperature $T_{c h}$ is about $0.156 \mathrm{GeV}$ [122], at which the hadron abundances are fixed. From the current results, the decrease of the pion mass around this temperature might contribute to the overpopulation of pions at low momenta. Also, the effect of pion splits at finite $\mu_{I}$ might contribute to the charge imbalance in the final particle spectra. Though the highest pion condensed temperature $T_{c, t o p}=$ $0.129 \mathrm{GeV}$ is a bit lower than $T_{c h}$, it is still higher than the thermal freeze-out temperature $T_{t h}$, which is estimated to be $0.1-0.12 \mathrm{GeV}$ [123-125]. Thus, the formation of pion condensate is possible before thermal freeze-out. Therefore, in the final detection, the low-energy pion and the coherent fraction would be enhanced. The quantitative relation between our results and the experimental data requires further study, and we leave this to future work.

\section{ACKNOWLEDGMENTS}

We would like to thank the useful discussion with Xinyang Wang and Lang Yu. H. L. is supported by the National Natural Science Foundation of China under Grant No. 11405074 . D. L. is supported by the National Natural Science Foundation of China under Grant No. 11805084, the PhD Start-up Fund of the Natural Science Foundation of Guangdong Province under Grant No. 2018030310457 , and the Guangdong Pearl River Talents Plan under Grant No. 2017 GC010480.

\section{APPENDIX: SHOOTING METHOD}

A second-order ODE can be numerically solved with two determining boundary conditions. However, in our work the boundary conditions always include free parameters. Therefore, we have to use the "shooting method" to

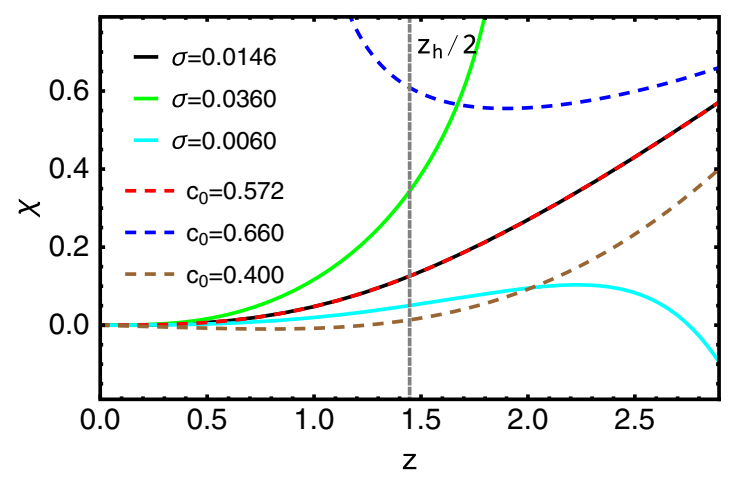

FIG. 11. Solutions for $\chi$ at $T=0.11 \mathrm{GeV}$. Different solutions are obtained with different values of $\sigma$ or $c_{0}$ with the UV boundary condition or the IR horizon boundary condition. The black solid line and the red dashed line overlap each other give the right solutions $\left(\sigma, c_{0}\right)=\left(0.0146 \mathrm{GeV}^{3}, 0.572 \mathrm{GeV}\right)$. 
numerically solve the EOMs. In this Appendix we use the EOM of $\chi$ in Eq. (13) as an example to illustrate how the "shooting method" works.

For the EOM of $\chi$ in Eq. (13), we have the expansion boundary conditions (14a) and (14b) for the UV boundary at $z=0$ with $\sigma$ undetermined and the IR horizon at $z=z_{h}$ with a free parameter $c_{0}$, respectively. On both sides, with a certain value of $\sigma$ or $c_{0}$, one can solve the equation numerically and get solutions for $\chi_{\sigma}$ or $\chi_{c_{0}}$ that are regular in the UV or IR, respectively. Requiring the smooth connection of $\chi_{\sigma}$ and $\chi_{c_{0}}$, one gets the two conditions $\chi_{\sigma}\left(z_{c}\right)=\chi_{c_{0}}\left(z_{c}\right)$ and $\chi_{\sigma}^{\prime}\left(z_{c}\right)=\chi_{c_{0}}^{\prime}\left(z_{c}\right)$, where $z_{c}$ is an arbitrary selected point in the interval $\left(0, z_{h}\right)$. However, we choose the midpoint $\left(z_{c}=z_{h} / 2\right)$ for convenience. Then, $\sigma$ and $c_{0}$ can be solved, and a full solution that is regular on both sides is obtained. Imposing such a "shooting" method, one can obtain the temperature-dependent chiral condensate and get information about the chiral phase transition. For the other EOMs, we just follow the illustrated "shooting method."

At last, we give a concrete example, shown in Fig. 11. At $T=0.110 \mathrm{GeV}$, if one lets the solutions from both sides satisfy $\chi_{\sigma}\left(z_{h} / 2\right)=\chi_{c_{0}}\left(z_{h} / 2\right)$ and $\chi_{\sigma}^{\prime}\left(z_{h} / 2\right)=\chi_{c_{0}}{ }^{\prime}\left(z_{h} / 2\right)$ simultaneously, the values $\sigma=0.0360 \mathrm{GeV}^{3}$ and $c_{0}=$ $0.660 \mathrm{GeV}$ are obtained. With these values, the solutions $\chi_{\sigma}(z)$ and $\chi_{c_{0}}(z)$ overlap each other, as shown by the solid black and dashed red lines. When the values of $\sigma$ or $\chi_{0}$ deviate from them, such as $\sigma=0.0146,0.006 \mathrm{GeV}^{3}$ and $c_{0}=0.572,0.400 \mathrm{GeV}$, the corresponding solutions deviated from the right solutions and will never smooth connect with the solution from the other side.
[1] J. Adams et al. (STAR Collaboration), Nucl. Phys. A757, 102 (2005).

[2] J. Cleymans, I. Kraus, H. Oeschler, K. Redlich, and S. Wheaton, Phys. Rev. C 74, 034903 (2006).

[3] A. Andronic, P. Braun-Munzinger, and J. Stachel, Phys. Lett. B 673, 142 (2009); 678, 516(E) (2009).

[4] A. Dumitru, U. Katscher, J. A. Maruhn, H. Stoecker, W. Greiner, and D. H. Rischke, Phys. Rev. C 51, 2166 (1995).

[5] B. B. Abelev et al. (ALICE Collaboration), Phys. Rev. C 89, 024911 (2014).

[6] V. Begun, W. Florkowski, and M. Rybczynski, Phys. Rev. C 90, 014906 (2014).

[7] V. Begun and W. Florkowski, Phys. Rev. C 91, 054909 (2015).

[8] M. Sako, T. Murakami, Y. Nakai, Y. Ichikawa, K. Ieki, S. Imajo, T. Isobe, M. Matsushita, J. Murata, and S. Nishimura et al., arXiv:1409.3322.

[9] M. Ishii, H. Kouno, and M. Yahiro, Phys. Rev. D 95, 114022 (2017).

[10] B. B. Brandt, A. Francis, H. B. Meyer, and D. Robaina, Phys. Rev. D 90, 054509 (2014).

[11] B. B. Brandt, A. Francis, H. B. Meyer, and D. Robaina, Phys. Rev. D 92, 094510 (2015).

[12] C. S. Fischer, Prog. Part. Nucl. Phys. 105, 1 (2019).

[13] F. Gao and M. Ding, arXiv:2006.05909.

[14] R. A. Tripolt, N. Strodthoff, L. von Smekal, and J. Wambach, Phys. Rev. D 89, 034010 (2014).

[15] Z. Wang and P. Zhuang, Phys. Rev. D 96, 014006 (2017).

[16] D. Ebert, Y. L. Kalinovsky, and M. K. Volkov, Phys. Lett. B 301, 231 (1993).

[17] T. Xia, L. He, and P. Zhuang, Phys. Rev. D 88, 056013 (2013).

[18] T. Xia, J. Hu, and S. Mao, Chin. Phys. C 43, 054103 (2019).

[19] J. Chao, M. Huang, and A. Radzhabov, Chin. Phys. C 44, 034105 (2020).
[20] H. Liu, X. Wang, L. Yu, and M. Huang, Phys. Rev. D 97, 076008 (2018).

[21] K. Xu, J. Chao, and M. Huang, arXiv:2007.13122.

[22] E. V. Shuryak, Phys. Rev. D 42, 1764 (1990).

[23] D. T. Son and M. A. Stephanov, Phys. Rev. Lett. 88, 202302 (2002).

[24] D. T. Son and M. A. Stephanov, Phys. Rev. D 66, 076011 (2002).

[25] J. M. Maldacena, Int. J. Theor. Phys. 38, 1113 (1999).

[26] S. S. Gubser, I. R. Klebanov, and A. M. Polyakov, Phys. Lett. B 428, 105 (1998).

[27] E. Witten, Adv. Theor. Math. Phys. 2, 253 (1998).

[28] P. Kovtun, D. T. Son, and A. O. Starinets, Phys. Rev. Lett. 94, 111601 (2005).

[29] D. Teaney, J. Lauret, and E. V. Shuryak, Phys. Rev. Lett. 86, 4783 (2001).

[30] P. Huovinen, P. F. Kolb, U. W. Heinz, P. V. Ruuskanen, and S. A. Voloshin, Phys. Lett. B 503, 58 (2001).

[31] T. Hirano, U. W. Heinz, D. Kharzeev, R. Lacey, and Y. Nara, Phys. Lett. B 636, 299 (2006).

[32] P. Romatschke and U. Romatschke, Phys. Rev. Lett. 99, 172301 (2007).

[33] A. Karch and E. Katz, J. High Energy Phys. 06 (2002) 043.

[34] J. Babington, J. Erdmenger, N. J. Evans, Z. Guralnik, and I. Kirsch, Phys. Rev. D 69, 066007 (2004).

[35] M. Kruczenski, D. Mateos, R. C. Myers, and D. J. Winters, J. High Energy Phys. 05 (2004) 041.

[36] T. Sakai and S. Sugimoto, Prog. Theor. Phys. 113, 843 (2005).

[37] T. Sakai and S. Sugimoto, Prog. Theor. Phys. 114, 1083 (2005).

[38] J. Erlich, E. Katz, D. T. Son, and M. A. Stephanov, Phys. Rev. Lett. 95, 261602 (2005).

[39] A. Karch, E. Katz, D. T. Son, and M. A. Stephanov, Phys. Rev. D 74, 015005 (2006). 
[40] G. F. de Teramond and S. J. Brodsky, Phys. Rev. Lett. 94, 201601 (2005).

[41] S. S. Gubser and A. Nellore, Phys. Rev. D 78, 086007 (2008).

[42] S. S. Gubser, A. Nellore, S. S. Pufu, and F. D. Rocha, Phys. Rev. Lett. 101, 131601 (2008).

[43] O. DeWolfe, S. S. Gubser, and C. Rosen, Phys. Rev. D 83, 086005 (2011).

[44] U. Gursoy and E. Kiritsis, J. High Energy Phys. 02 (2008) 032.

[45] U. Gursoy, E. Kiritsis, and F. Nitti, J. High Energy Phys. 02 (2008) 019.

[46] O. Aharony, S. S. Gubser, J. M. Maldacena, H. Ooguri, and Y. Oz, Phys. Rep. 323, 183 (2000).

[47] J. Erdmenger, N. Evans, I. Kirsch, and Threlfall, Eur. Phys. J. A 35, 81 (2008).

[48] G. F. de Teramond and S. J. Brodsky, arXiv:1203.4025.

[49] A. Adams, L. D. Carr, T. Schäfer, P. Steinberg, and J. E. Thomas, New J. Phys. 14, 115009 (2012).

[50] S. J. Brodsky, G. F. de Teramond, H. G. Dosch, and J. Erlich, Phys. Rep. 584, 1 (2015).

[51] T. Gherghetta, J. I. Kapusta, and T. M. Kelley, Phys. Rev. D 79, 076003 (2009).

[52] T. M. Kelley, S. P. Bartz, and J. I. Kapusta, Phys. Rev. D 83, 016002 (2011).

[53] Y. Q. Sui, Y. L. Wu, Z. F. Xie, and Y. B. Yang, Phys. Rev. D 81, 014024 (2010).

[54] Y. Q. Sui, Y. L. Wu, and Y. B. Yang, Phys. Rev. D 83, 065030 (2011).

[55] D. Li, M. Huang, and Q. S. Yan, Eur. Phys. J. C 73, 2615 (2013).

[56] D. Li and M. Huang, J. High Energy Phys. 11 (2013) 088.

[57] P. Colangelo, F. De Fazio, F. Giannuzzi, F. Jugeau, and S. Nicotri, Phys. Rev. D 78, 055009 (2008).

[58] A. Ballon-Bayona and L. A. H. Mamani, Phys. Rev. D 102, 026013 (2020).

[59] E. F. Capossoli, M. A. M. Contreras, D. Li, A. Vega, and H. Boschi-Filho, Chin. Phys. C 44, 064104 (2020).

[60] M. Á. M. Contreras, A. Vega, and S. Cortés, Chin. J. Phys. 66, 715 (2020).

[61] P. Colangelo, F. Giannuzzi, S. Nicotri, and V. Tangorra, Eur. Phys. J. C 72, 2096 (2012).

[62] D. Dudal, D. R. Granado, and T. G. Mertens, Phys. Rev. D 93, 125004 (2016).

[63] K. Chelabi, Z. Fang, M. Huang, D. Li, and Y. L. Wu, Phys. Rev. D 93, 101901 (2016).

[64] K. Chelabi, Z. Fang, M. Huang, D. Li, and Y. L. Wu, J. High Energy Phys. 04 (2016) 036.

[65] Z. Fang, S. He, and D. Li, Nucl. Phys. B907, 187 (2016).

[66] D. Li, M. Huang, Y. Yang, and P. H. Yuan, J. High Energy Phys. 02 (2017) 030.

[67] D. Li and M. Huang, J. High Energy Phys. 02 (2017) 042.

[68] S. P. Bartz and T. Jacobson, Phys. Rev. D 94, 075022 (2016).

[69] Z. Fang, Y. L. Wu, and L. Zhang, Phys. Lett. B 762, 86 (2016).

[70] S. P. Bartz and T. Jacobson, Phys. Rev. C 97, 044908 (2018).

[71] Z. Fang, Y. L. Wu, and L. Zhang, Phys. Rev. D 98, 114003 (2018).
[72] K. Ghoroku and M. Yahiro, Phys. Rev. D 73, 125010 (2006).

[73] L. X. Cui and Y. L. Wu, Mod. Phys. Lett. A 28, 1350132 (2013).

[74] L. X. Cui, Z. Fang, and Y. L. Wu, Chin. Phys. C 40, 063101 (2016).

[75] Y. Nambu and G. Jona-Lasinio, Phys. Rev. 122, 345 (1961).

[76] Y. Nambu and G. Jona-Lasinio, Phys. Rev. 124, 246 (1961).

[77] M. Asakawa, T. Hatsuda, and Y. Nakahara, Prog. Part. Nucl. Phys. 46, 459 (2001).

[78] T. Yamazaki et al. (CP-PACS Collaboration), Phys. Rev. D 65, 014501 (2001).

[79] M. Laine, J. High Energy Phys. 05 (2007) 028.

[80] Y. Nakahara, M. Asakawa, and T. Hatsuda, Phys. Rev. D 60, 091503 (1999).

[81] G. D. Moore and O. Saremi, J. High Energy Phys. 09 (2008) 015.

[82] D. Teaney, Phys. Rev. D 74, 045025 (2006).

[83] G. Aarts and J. M. M. Resco, J. High Energy Phys. 04 (2002) 053.

[84] P. Kovtun and A. Starinets, Phys. Rev. Lett. 96, 131601 (2006).

[85] G. Aarts, C. Allton, J. Foley, S. Hands, and S. Kim, Phys. Rev. Lett. 99, 022002 (2007).

[86] M. Fujita, K. Fukushima, T. Misumi, and M. Murata, Phys. Rev. D 80, 035001 (2009).

[87] A. Jakovac, P. Petreczky, K. Petrov, and A. Velytsky, Phys. Rev. D 75, 014506 (2007).

[88] P. Colangelo, F. Giannuzzi, and S. Nicotri, Phys. Rev. D 80, 094019 (2009).

[89] P. Colangelo, F. Giannuzzi, and S. Nicotri, J. High Energy Phys. 05 (2012) 076.

[90] M. M. Aggarwal et al. (STAR Collaboration), arXiv: 1007.2613.

[91] G. Odyniec, Proc. Sci., CPOD2013 (2013) 043.

[92] X. Luo and N. Xu, Nucl. Sci. Tech. 28, 112 (2017).

[93] D. Albrecht and J. Erlich, Phys. Rev. D 82, 095002 (2010).

[94] B. H. Lee, S. Mamedov, S. Nam, and C. Park, J. High Energy Phys. 08 (2013) 045.

[95] H. Nishihara and M. Harada, Phys. Rev. D 89, 076001 (2014).

[96] H. Nishihara and M. Harada, Phys. Rev. D 90, 115027 (2014).

[97] S. Mamedov, Eur. Phys. J. C 76, 83 (2016).

[98] M. Lv, D. Li, and S. He, J. High Energy Phys. 11 (2019) 026.

[99] X. Cao, H. Liu, D. Li, and G. Ou, Chin. Phys. C 44, 083106 (2020).

[100] D. M. Rodrigues, D. Li, E. Folco Capossoli, and H. Boschi-Filho, Europhys. Lett. 128, 61001 (2019).

[101] D. M. Rodrigues, D. Li, E. F. Capossoli, and H. BoschiFilho, Phys. Rev. D 98, 106007 (2018).

[102] S. I. Finazzo, R. Rougemont, H. Marrochio, and J. Noronha, J. High Energy Phys. 02 (2015) 051.

[103] R. Rougemont, R. Critelli, J. Noronha-Hostler, J. Noronha, and C. Ratti, Phys. Rev. D 96, 014032 (2017).

[104] R. Zöllner and B. Kämpfer, Eur. Phys. J. Plus 135, 304 (2020).

[105] D. Li, S. He, M. Huang, and Q. S. Yan, J. High Energy Phys. 09 (2011) 041. 
[106] R. G. Cai, S. He, and D. Li, J. High Energy Phys. 03 (2012) 033.

[107] X. Chen, D. Li, D. Hou, and M. Huang, J. High Energy Phys. 03 (2020) 073.

[108] S. He, Y. Yang, and P. H. Yuan, arXiv:2004.01965.

[109] A. Ballon-Bayona, H. Boschi-Filho, E. F. Capossoli, and D. M. Rodrigues, arXiv:2006.08810 [Phys. Rev. D (to be published)].

[110] L. A. H. Mamani, C. V. Flores, and V. T. Zanchin, Phys. Rev. D 102, 066006 (2020).

[111] H. Boschi-Filho, N. R. F. Braga, F. Jugeau, and M. A. C. Torres, Eur. Phys. J. C 73, 2540 (2013).

[112] E. F. Capossoli, D. Li, and H. Boschi-Filho, Eur. Phys. J. C 76, 320 (2016).

[113] A. Cherman, T. D. Cohen, and E. S. Werbos, Phys. Rev. C 79, 045203 (2009).

[114] J. Chen, S. He, M. Huang, and D. Li, J. High Energy Phys. 01 (2019) 165.
[115] D. T. Son and A. O. Starinets, J. High Energy Phys. 09 (2002) 042.

[116] Z. Abidin and C. E. Carlson, Phys. Rev. D 77, 115021 (2008).

[117] Z. Abidin and P. T. P. Hutauruk, Phys. Rev. D 100, 054026 (2019).

[118] X. Cao, S. Qiu, H. Liu, and D. Li (to be published).

[119] B. A. Li, C. M. Ko, and W. Bauer, Int. J. Mod. Phys. E 07, 147 (1998).

[120] D. T. Son and M. A. Stephanov, Phys. Rev. Lett. 86, 592 (2001).

[121] B. B. Brandt, G. Endrodi, and S. Schmalzbauer, Phys. Rev. D 97, 054514 (2018).

[122] J. Stachel, A. Andronic, P. Braun-Munzinger, and K. Redlich, J. Phys. Conf. Ser. 509, 012019 (2014).

[123] S. Pratt and K. Haglin, Phys. Rev. C 59, 3304 (1999).

[124] I. Melo and B. Tomasik, J. Phys. G 43, 015102 (2016).

[125] D. Prorok, J. Phys. G 43, 055101 (2016). 